Pacific Journal of Mathematics

PPROXIMATING EQUIVARIANT MAPPING SPACES

R. Costenoble, Stefan Wanker and G. S. Wells 


\title{
APPROXIMATING EQUIVARIANT MAPPING SPACES
}

\author{
S. R. Costenoble, S. Waner, And G. S. Wells
}

\begin{abstract}
Let $S V$ and $S^{V}$ be the unit sphere and one-point compactification of the unitary representation $V$ of the finite group $G$. One has the associated self-mapping $G$-spaces $\mathscr{U}(S V, S V)$ and $\Omega^{V} S^{V}$ respectively, the first consisting of unbased maps and the second of based maps. It is the goal of this paper to describe homotopy approximations of these loop spaces (as examples of a more general class of $G$-spaces), along the lines of the group completion approximations of Segal, McDuff and Hauschild. We then apply these approximations to obtain splittings and Hopf space structures for several spaces.
\end{abstract}

Introduction. Let $G$ be a finite group and let $V$ be a finite dimensional unitary representation of $G$ with unit sphere $S V$. Let $\mathscr{U}(S V, S V)$ be the $G$-space of unbased maps $S V \rightarrow S V$, where $G$ acts by conjugation. If $S^{V}$ is the one point compactification of $V$, denote the $G$-space of based maps $S^{V} \rightarrow S^{V}$ by $\Omega^{V} S^{V}$. It is the goal of this paper to describe properties of these loop spaces, viewed as examples of a more general class of $G$-spaces, through the use of $G$-homotopy approximations.

May [M1] first used configurations of "little cubes" to obtain a nonequivariant homotopy approximation of $\Omega^{n} \Sigma^{n} Z$ for a connected based CW complex $Z$. He also showed that the little cubes construction can be replaced by an analogous configuration space construction. Segal [S] then showed that $\Omega^{n} S^{n}\left(=\Omega^{n} \Sigma^{n} S^{0}\right)$ is the group completion of the space of configurations of points in the unit disc $D^{n}$. Formally, a group completion is a map $\alpha: X \rightarrow Y$ from a Hopf space to a grouplike Hopf space such that $\alpha$ coincides with localization at $\pi_{0}(X)$ in homology with field coefficients. May's use of configurations of little cubes rather than points had the effect of greatly simplifying the arguments, although the corresponding approximating spaces are homotopy equivalent. McDuff [M2] generalized Segal's result to obtain approximations up to group completion of spaces of sections of certain sphere bundles over a compact manifold $M$. Generalizing May's result, Caruso and Waner [CW1] showed that $\Omega^{n} \Sigma^{n} Z$ is homotopy equivalent to a space of configurations of "positive and negative little cubes" in the $n$-disc $D^{n}$, whether or not $Z$ is connected. (McDuff 
had shown in [M2] that the space of configurations of positive and negative particles is not homotopy equivalent to $\Omega^{n} S^{n}$.)

Hauschild $[\mathbf{H}]$ obtained an equivariant analogue of McDuff's results: If the real orthogonal representation $V$ has a trivial summand, then $\Omega^{V} S^{V}$ is an equivariant group completion of the space of configurations of points in the unit disc of $V$. Here, an equivariant group completion is a $G$-map of Hopf spaces that respects structure and restricts to a group completion on each fixed subset. The Hopf space structure in $\Omega^{V} S^{V}$ is defined using addition of maps in the standard way.

When the representation $V$ possesses no trivial summand, the corresponding unbased $G$-space $\mathscr{U}(S V, S V)$ possesses no Hopf space structure corresponding to addition, and the notion of a group completion makes no sense. However, one may still ask whether this space admits a homotopy approximation by a simpler $G$-space.

As indicated above, the results in this paper provide $G$-homotopy approximations for the unbased spaces $\mathscr{U}(S V, S V)$ when $V$ is a unitary representation of $G$, and for $\Omega^{V} S^{V}$ when $V$ is a real orthogonal representation with a trivial summand. We give two applications. First, we obtain splittings in unstable based and unbased equivariant homotopy, along the lines of tom Dieck [D]. Second, we prove that $\operatorname{colim}_{V} \mathscr{U}(S V, S V)$ possesses an equivariant Hopf space structure compatible with addition of homotopy classes, the colimit being taken over a suitable collection of unitary representations $V$ with $V^{G}=0$. In addition, we show that this Hopf-space structure comes from an equivariant infinite loop space structure.

Our approximation results occur as special cases of a more general result along the lines of McDuff's work (as generalized by Hauschild), but without any assumption that the $G$-manifolds in question have boundaries, and providing approximations up to homotopy rather than up to group completion. Specifically, let $M$ be a compact $G$ manifold with or without boundary, let $N$ be a closed codimensionzero submanifold of $\partial M$, and let $F\left(\tau_{M}\right)$ be the fiberwise one-point compactification of the tangent bundle of $M$. Denoting fiberwise smash product by $\wedge_{F}$, let $\Gamma(M, N ; X)$ be the space of all sections of $F\left(\tau_{M}\right) \wedge_{F} X \rightarrow M$ which coincide with the trivial section on $\operatorname{cl}(\partial M-N)$. The spaces we construct are approximations of $\Gamma(M, N ; X)$ up to $G$-homotopy when $X$ is a $G$-CW complex; these are spaces of configurations of "PL-arcs" in $M$ lying along a specified "direction". To make sense of the idea of a specified direction, we 
assume that $M$ is fibered by oriented 1-manifolds. The precise construction of the approximationg spaces and the statement of the main theorem are given in $\S 1 . \S 2$ gives the applications, and the remaining sections are devoted to the proof of our main result.

The authors are indebted to Hofstra University and to the University of the Witwatersrand for having provided released time.

1. PL-arcs. We shall be considering smooth compact $G$-manifolds whose components are either principal $\left(G, S^{1}\right)$-bundles, or of the form $M^{\prime} \times I$ with trivial $G$-action on $I$. These can be considered together as essentially the only examples of smooth compact $G$ manifolds that are PL-bundled by oriented 1-manifolds. Precisely, we assume given an equivariant PL-bundle $\pi: M \rightarrow M^{\prime}$ with fiber a 1-manifold $Y$ and structure group reduced to PL $\cap$ Diff. We also assume given a trivialization of the bundle of tangents along the fibers, and we assume that the action of $G$ preserves this orientation of the fibers. These restrictions are needed to make sense of the geometric constructions we consider. Possibly changing $M^{\prime}$, we may conclude that $M \rightarrow M^{\prime}$ is a union of components, each of which is either of the form $M^{\prime \prime} \times I \rightarrow M^{\prime \prime}$, or is a principal $\left(G, S^{1}\right)$-bundle.

We shall also assume given $G$-invariant Riemannian metrics on $M$ and $M^{\prime}$, with the following relation: If $M=M^{\prime} \times I$, then the metric on $M$ should be the product of the metric on $M^{\prime}$ and the standard metric on $I$. On the other hand, if $M$ is a principal $\left(G, S^{1}\right)$-bundle, so that $G \times S^{1}$ acts on $M$ with the action of $S^{1}$ being free, then we assume that $M$ has a $G \times S^{1}$-invariant metric and that $M^{\prime}=M / S^{1}$ has the $G$-invariant metric given in the tangents perpendicular to the fibers in $M$. In general, $M$ will be a union of components with metrics of this kind.

Two examples to bear in mind are the projection $S V \rightarrow S V / S^{1}$ for a unitary $G$-module $V$, and the projection $M^{\prime} \times I \rightarrow M^{\prime}$ for any smooth $G$-manifold $M^{\prime}$. The first of these will lead to the desired approximations of spaces of self-maps of $S V$, while the second will be required in the induction arguments involved in analyzing the first case. Although it would perhaps be convenient to concentrate on one case only, the way in which the cases interlock in the induction, as well as the fundamental ways in which the arguments for the two cases differ, make this unfeasible.

In view of the very restricted structure, it makes sense to talk about a PL-map from a fiber of $\pi$ into $S^{1}$, and to talk about the sign of the slope of any of the linear segments of such a map. Also, for any 
component $Y$ of any fiber, there is a unique orientation-preserving linear diffeomorphism of $Y$ with either $I$ or $S^{1}$. Unless otherwise specified we let $I=[-1,1]$, and $S^{1}=I /\{-1,1\}$ with basepoint $e=\{-1,1\}$.

Definitions 1.1. A PL-arc in the G-manifold $M$ is a PL-map from one of its fibers into $S^{1}$, so that each of its nontrivial linear segments (that is, segments whose value is not constant at the basepoint $e$ ) has nonzero slope. The support, $\operatorname{supp} \nu$, of a PL-arc $\nu$ in $M$ is $\nu^{-1}\left(S^{1}-e\right)$; two PL-arcs $\nu$ and $\mu$ in $M$ are disjoint if their supports are disjoint. If two disjoint PL-arcs $\nu$ and $\mu$ are defined on the same fiber, we define $\nu \oplus \mu$ to be their pointwise product in $S^{1}$ (i.e., their superposition). An indecomposable PL-arc in $M$ is an arc that cannot be expressed as a sum of two nontrivial PL-arcs. Finally, we refer to a PL-arc $\nu$ in $M$ as closed if $\nu \mid \partial M$ is trivial.

We topologize the set $\operatorname{Arc}(M)$ of PL-arcs in $M$ as a $G$-bundle over $M^{\prime}$ with fiber the space $\operatorname{PL}\left(Y, S^{1}\right)$ of PL-maps $Y \rightarrow S^{1}$. For $n \geq 0$, let $L(M)(n)$ be the $G$-space of $n$-tuples of pairwise disjoint closed PL-arcs in $M$, topologized as a subspace of $\operatorname{arc}(M)^{n}$. Note that $L(M)(n)$ is acted on freely by the symmetric troup $\Sigma_{n}$, this action commuting with the $G$-action.

Now let $X$ be a nondegenerately based $G$-space with stationary basepoint $*$, and define

$$
L(M, X)=\coprod_{n \geq 0} L(M)(n) \times \Sigma_{\Sigma_{n}} X^{n} / \approx
$$

where $\left[\nu_{1}, \ldots, \nu_{n} ; x_{1}, \ldots, x_{n}\right] \approx\left[\nu_{2}, \ldots, \nu_{n} ; x_{2}, \ldots, x_{n}\right]$ if either $\nu_{1}$ is trivial or $x_{1}=*$, and

$$
\left[\nu_{1}, \ldots, \nu_{n} ; x_{1}, \ldots, x_{n}\right] \approx\left[\nu_{1} \oplus \nu_{2}, \ldots, \nu_{n} ; x_{2}, \ldots, x_{n}\right]
$$

if $x_{1}=x_{2}$ and $\nu_{1} \oplus \nu_{2}$ is defined. $L(M ; X)$ is a $G$-space with the diagonal $G$-action. Write $L(M)$ for $L\left(M ; S^{0}\right)$.

For a relative version, let $N$ be a $G$-invariant codimension-zero submanifold of $\partial M$, and define

$$
L(M, N ; X)=\coprod_{n \geq 0} L(M, N)(n) \times_{\Sigma_{n}} X^{n} / \approx .
$$

Here $L(M, N)(n)$ is defined in a way similar to $L(M)(n)$, except that one allows PL-arcs with support in $(M-\partial M) \cup N$ rather than only in $M-\partial M$, and one uses the additional equivalence relation given by identifying $\left[\nu_{1}, \ldots, \nu_{n} ; x_{1}, \ldots, x_{n}\right]$ with $\left[\nu_{2}, \ldots, \nu_{n} ; x_{2}, \ldots, x_{n}\right]$ 
if the support of $\nu_{1}$ lies in $N$, so that PL-arcs in $M$ "disappear" when they enter $N$. We shall denote $L\left(M, N ; S^{0}\right)$ by $L(M, N)$.

We now impose a restriction on the submanifold $N$. First observe that $\partial M$ decomposes as a union $\partial M_{1} \cup \partial M_{2}$, where $\partial M_{1}=$ $\pi^{-1}\left(\partial M^{\prime}\right)$ and $\partial M_{2}$ is the space of endpoints of the fibers. We assume from now on that $N=N_{1} \cup N_{2}$, where $N_{1}=\pi^{-1}\left(N^{\prime}\right)$ for a codimension-zero submanifold $N^{\prime} \subset \partial M^{\prime}$ and $N_{2}$ is a union of components of $\partial M_{2}$. Thus, if $N$ contains any point of a fiber other than an endpoint, it must contain the whole fiber.

We also need to use the classical configuration spaces (cf. $[\mathbf{H}])$. For $n \geq 0$ let $C(M)(n)$ be the $G$-space of $n$-tuples of distinct points in $M-\partial M$, and if $N \subset M$ define $C(M, N)(n)$ as the configuration space analogue of $L(M, N)(n)$. The construction above then yields a $G$-space $C(M, N ; X)$. When discussing configuration space models, we make no assumptions about any fiberings of $M$.

We can now state the main theorem. Let $\tau_{M}$ denote the tangent bundle of $M$. Let $F\left(\tau_{M}\right)$ denote the fiberwise one-point compactification of $\tau_{M}$. If $\wedge_{\mathscr{F}}$ is fiberwise smash product, let $\Gamma(M, N ; X)$ be the space of all sections of $F\left(\tau_{M}\right) \wedge_{\mathscr{F}} X \rightarrow M$ that coincide with the basepoint section on $\operatorname{cl}(\partial M-N)$.

THEOREM 1.2. (a) If $X$ is a nondegenerately based $G$-space, then $\Gamma(M, N ; X)$ is weakly G-equivalent to $L(M, N ; X)$. Further, if $X$ has the G-homotopy type of a $G$-CW complex, then there exists a $G$ homotopy equivalence

$$
\gamma: L(M, N ; X) \rightarrow \Gamma(M, N ; X) .
$$

(b) If $X$ is a nondegenerately based $G$-space with connected fixed-sets, then the conclusion of (a) continues to hold with $L(M, N ; X)$ replaced by $C(M, N ; X)$.

(c) For arbitrary $X$, there exists a map

$$
\delta: C(M, N ; X) \rightarrow L(M, N ; X)
$$

such that $\gamma \delta \simeq \gamma$ as G-maps.

Note that if $X$ has the $G$-homotopy type of a $G$-CW complex, then both spaces in question have the $G$-homotopy type of $G-\mathrm{CW}$ complexes. Thus the conclusion that $\gamma$ is a $G$-homotopy equivalence follows by the equivariant Whitehead Theorem. (See, for example, [W].) 
A consequence of the theorem is that it provides equivariant homotopy approximations for the spaces $\mathscr{U}(S V, S V)$ discussed in the introduction: Let $V$ be any finite dimensional unitary representation of $G$ with unit sphere $S V$. The $G$-space $\mathscr{U}(S V, S V)$ is then $G$-homeomorphic with $\Gamma\left(S V, \varnothing ; S^{0}\right)$, since $F\left(\tau_{S V}\right)$ is the trivial $G$ bundle $S V \times S V \rightarrow S V$. We now have

COROLlARY 1.3. With $V$ as above, there exists a G-homotopy equivalence

$$
\gamma: L(S V) \rightarrow \mathscr{U}(S V, S V)
$$

In fact we shall see that the approximations $\gamma$ are natural in $V$ up to $G$-homotopy, as a consequence of the explicit constructions below.

In order to deduce approximations for mapping spaces of based spheres along the lines of the group completion results of Hauschild [H], we take $M=D V \times I \cong D(V \oplus \mathbb{R})$ for $V$ any real orthogonal representation of $G$, and $N=\partial M . \Gamma(M, N ; X)$ is then the equivariant loop space $\Omega^{W} \Sigma^{W} X$ of based maps $S^{W} \rightarrow X \wedge S^{W}$ where $W=V \oplus \mathbb{R}$. We then obtain the following:

COROLlARY 1.4. If $W$ contains a trivial summand, there exists a G-homotopy equivalence

$$
\gamma: L(D W, S W ; X) \rightarrow \Omega^{W} \Sigma^{W} X .
$$

2. Applications. Before proving Theorem 1.2, we give some applications.

Let $M$ be a $G$-manifold, and let $H$ be a subgroup of $G$. Let $\widetilde{M}_{H}$ be the complement in $M^{H}$ of a regular neighborhood of $\bigcup_{K \supsetneqq H} M^{K}$. Then $\widetilde{M}_{H}$ is a free compact $W H=N H / H$-manifold. Let $\Gamma_{G}$ denote the space of $G$-invariant sections.

Theorem 2.1 (Splitting of Unstable Equivariant Mapping Spaces). There is a weak equivalence

$$
\Gamma_{G}(M, N ; X) \simeq \prod_{(H)} \Gamma\left(E W H \times_{W H} \widetilde{M}_{H}, E W H \times_{W H} \widetilde{N}_{H} ; X^{H}\right) .
$$

The product runs over all conjugacy classes of subgroups of $G$, and the spaces appearing on the right are nonequivariant spaces of sections of the bundles

$$
E W H^{+} \wedge_{W H} F\left(\tau_{\widetilde{M}_{H}}\right) \wedge_{\mathscr{F}} X^{H} \rightarrow \widetilde{M}_{H} / W H
$$


Proof. Denote the $G$-fixed subspace of $L(M, N ; X)$ by $L_{G}(M, N ; X)$. Then Theorem 1.2 says that $\Gamma_{G}(M, N ; X)$ is equivalent to $L_{G}(M, N ; X)$. If $H$ is a subgroup of $G$ with conjugacy class $(H)$, let $L_{G}(M, N ; X)_{(H)}$ be the subspace made up of arcs with isotropy in $(H)$. It is easy to see that we have a decomposition

$$
L_{G}(M, N ; X) \simeq \prod_{(H)} L_{G}(M, N ; X)_{(H)} .
$$

This is true because we are looking at the $G$-invariant configurations, and in these the orbit type is locally constant. Note further that each factor $L_{G}(M, N ; X)_{(H)}$ is homotopy equivalent to $L_{W H}\left(\widetilde{M}_{H}, \widetilde{N}_{H} ; X^{H}\right)$.

Again, $L_{W H}\left(\widetilde{M}_{H}, \widetilde{N}_{H} ; X^{H}\right) \simeq \Gamma_{W H}\left(\widetilde{M}_{H}, \widetilde{N}_{H} ; X^{H}\right)$. By well-known arguments, the $W H$-invariant sections of $F\left(\tau_{\widetilde{M}_{H}}\right) \wedge \mathscr{F} X^{H} \rightarrow \widetilde{M}_{H}$ are in 1-1 correspondence with the nonequivariant sections of

$$
E W H^{+} \wedge_{W H} F\left(\tau_{\widetilde{M}_{H}}\right) \wedge_{\mathscr{F}} X^{H} \rightarrow \widetilde{M}_{H} / W H,
$$

and so the theorem follows.

In the special case that each component of each fixed set of $M$ intersects $N$, the methods of Hauschild [H] can be used to obtain the splitting. This connectivity condition is necessary in order to apply his methods, because, without it, his quasifibration arguments would fail.

In view of Corollary 1.3, we obtain splittings of the space $\mathscr{U}(S V, S V)^{G}$ of $G$-maps $S V \rightarrow S V$ when $V$ is unitary. The splitting of $\left(\Omega^{W} \Sigma^{W} X\right)^{G}$ that we get from Corollary 1.4 (where $W$ is a real representation with a trivial summand) was known to Hauschild [H].

If $V$ is unitary with nontrivial $G$-fixed set, then $\pi_{0}\left(\mathscr{U}(S V, S V)^{G}\right)$ possesses a group structure given by addition of homotopy classes in the usual way. When $V^{G}=0$, one may again obtain such a structure by appealing to the general position arguments in [WW]. This addition does not arise from a Hopf space structure on $\mathscr{U}(S V, S V)$, since such a structure fails to exist even in the nonequivariant case. Passing to the stable case nonequivariantly, one has $\operatorname{colim}_{n} \mathscr{U}\left(S^{n}, S^{n}\right) \simeq$ colim $\Omega^{n} S^{n}$, resulting in a Hopf space structure on the former. In the equivariant case, we now have the following consequence of Theorem 1.2. Let $U$ be a countably infinite direct sum of finite dimensional unitary $G$-modules, such that $U \cong U \oplus U$; we are particularly interested in the case $U^{G}=0$. 
THEOREM 2.2. The G-space $\operatorname{colim}_{V \subset U} \mathscr{U}(S V, S V)$ has a homotopy associative and commutative equivariant Hopf space structure such that the restrictions

$$
\operatorname{colim}_{V \subset U} \mathscr{U}(S V, S V)^{K} \rightarrow \operatorname{colim}_{V \subset U} \mathscr{U}\left(S V^{K}, S V^{K}\right)
$$

for $K \subset G$ agree with the additive Hopf space structure on $\operatorname{colim}_{n} \mathscr{U}\left(S^{n}, S^{n}\right)$. In fact, $\operatorname{colim}_{V \subset U} \mathscr{U}(S V, S V)$ is G-homotopy equivalent to an equivariant infinite loop space.

Proof. We first give the Hopf-space structure directly. By Corollary 1.3 and naturality, we may replace $\operatorname{colim}_{V \subset U} \mathscr{U}(S V, S V)$ by $\operatorname{colim}_{V \subset U} L(S V) \simeq L\left(\operatorname{colim}_{V \subset U} S V\right)$. Here, $L\left(\operatorname{colim}_{V \subset U} S V\right)$ is defined in the same way as for finite dimensional $G$-manifolds. The $G$-space $E=\operatorname{colim}_{V \subset U} S V$ is in fact a model for the universal $G$ space $E \mathscr{F}$, where $\mathscr{F}$ is the family $\left\{K \subset G: U^{K} \neq 0\right\}$; however, we do not use this fact explicitly. The desired Hopf space structure is a composite

$$
L(E) \times L(E) \rightarrow L(E \amalg E) \rightarrow L(E) .
$$

Here, the first arrow is the assembly map. The second arrow is induced by the $G$-inclusion $E \amalg E \rightarrow E$ obtained from

$$
U \amalg U \stackrel{\Delta}{\longrightarrow} U \oplus U \cong U,
$$

where $\Delta$ takes the first copy of $U$ into $U \oplus 0$, and the second copy into $0 \oplus U$. Since $L$ is functorial on inclusions, this gives a well-defined map $L(E \amalg E) \rightarrow L(E)$.

To show that $L(E)$ is equivalent to an equivariant infinite loop space, we appeal to [CW3]. To use the results from that paper, we need only observe that the construction of the multiplication above generalizes to an action by the equivariant linear isometries operad (described in [CW3]).

REMARKs 2.3. (a) Let $\mathscr{U}$ be as in Theorem 2.2. Then the approximating map $\gamma: \operatorname{colim}_{V \subset U} C(S V) \rightarrow \operatorname{colim}_{V \subset U} \mathscr{U}(S V, S V)$ ought to be a group completion. Unfortunately, we do not have a rigorous proof, although we believe that the following sketch could be made rigorous with the addition of some long and tedious arguments.

Sketch of Proof. By Theorem 1.2(c) one has a $G$-map $\delta: C(S V) \rightarrow$ $L(S V)$. By the proof of Theorem 2.1, the fixed-sets of both spaces split as products of nonequivariant configuration spaces or arc spaces of manifolds $\widetilde{M}(V)_{H} / N H$. We shall see as a consequence of the 
constructions below that this splitting is compatible with the map $\delta$. Further, the constructions also show that on each factor, the restriction of $\delta$ agrees with the classical approximation map of McDuff [M2] when we compose with the approximating map $\gamma$.

When $\widetilde{M}(V)_{H} / N H$ has boundary, the corresponding map, although not a map between Hopf-spaces, behaves formally like a group completion in the sense of [M2]. Explicitly, taking the colimit of the configuration spaces under translation maps gives a homology equivalence. (This idea is used again in $[\mathbf{H}]$.) This property is preserved by the taking of colimits over $V \subset \mathscr{U}$, and here the McDuff construction agrees in homology with group completion under the Hopf-space structure given in Theorem 2.2.

When $\widetilde{M}(V)_{H} / N H$ is closed, we must first pass to the colimit over $V \subset \mathscr{U}$, and replace $\operatorname{colim}_{V \subset U} \widetilde{M}(V)_{H} / N H$ by a corresponding colimit obtained by deleting a small disc. This procedure causes no harm to the argument, as the corresponding configurations and function spaces fit into quasifibrations with common base space $\widetilde{M}(V)_{H} / N H$. We can use the Serre spectral sequence to compare the homologies.

Finally, since group completion is preserved by products, the result should follow.

(b) In the event that the manifolds $M$ and $N$ satisfy the connectivity requirements of Hauschild $[\mathbf{H}]$, so that $C(M, N ; X)$ has any hope at all of being a Hopf-space, his original arguments, which were stated only for the case $X=S^{0}$, easily generalize to show that

$$
\gamma: C(M, N ; X) \rightarrow \Gamma(M, N ; X)
$$

is a group completion when $C(M, N ; X)$ and $\Gamma(M, N ; X)$ are Hopf-spaces.

3. Construction of the approximating map. The approximating maps $\gamma$ are not defined directly on the spaces $L(M, N ; X)$, but are defined instead on certain equivalent spaces, which we now construct.

If $\nu$ is a PL-arc in $M$ and if $\varepsilon>0$, then $\nu$ determines associated cylinders $C(\nu, \varepsilon)$ and $\bar{C}(\nu, \varepsilon)$ of radius $\varepsilon$ as follows. Say that two points $m$ and $m^{\prime}$ of $M$ are normally equivalent if there is an arc from $m$ to $m^{\prime}$ covering a minimal geodesic in $M^{\prime}$ and normal to every fiber it intersects. Given the geodesic in $M^{\prime}$, such an arc is unique. Denote $\pi(\operatorname{supp} \nu)$ by $[\nu]$. With $d_{M^{\prime}}$ the metric on $M^{\prime}$, set

$$
C(\nu, \varepsilon)=\{x \in M ; x \text { normally equivalent to some point }
$$


and

$$
\begin{aligned}
& \bar{C}(\nu, \varepsilon)=\{x \in M ; x \text { normally equivalent to some point } \\
& \left.\qquad \text { in } \operatorname{cl}(\operatorname{supp} \nu) \text { and } d_{M^{\prime}}(\pi(x),[\nu]) \leq \varepsilon\right\},
\end{aligned}
$$

For $\varepsilon$ sufficiently small and $\operatorname{supp} \nu$ interior to $M$, these are diffeomorphic copies of the standard cylinders $\left(D^{n-1}\right)^{\prime} \times \operatorname{supp} \nu$ and $D^{n-1} \times$ $\operatorname{cl}(\operatorname{supp} \nu)$ respectively. This will be discussed further below. We say that the cylinder $C(\nu, \varepsilon)$ overlaps the subspace $N \subset M$ if $C(\nu, \varepsilon) \cap N$ $\neq \varnothing$.

Now define a subspace $L(M, N ; X)(\varepsilon)$ of $L(M, N ; X)$ by considering only those tuples of PL-arcs $\nu$ whose associated open cylinders of radius $\varepsilon$ are pairwise non-overlapping and do not overlap $\partial M-N$. Fix some $\varepsilon_{0}>0$ and let

$$
\mathscr{L}(M, N ; X)=\bigcup_{0<\varepsilon \leq \varepsilon_{0}} L(M, N ; X)(\varepsilon) \times\{\varepsilon\},
$$

topologized as a subspace of $L(M, N ; X) \times \mathbb{R}$.

Configuration space models are defined similarly and more simply; take $C(M, N ; X)(\varepsilon)$ to be the subspace of $C(M, N ; X)$ using configurations of points at least $2 \varepsilon$ apart and also at least a distance $\varepsilon$ from $\partial M-N$. Then define $\mathscr{C}(M, N ; X)$ in the analogous way. Recall that here we assume no fibered structure on $M$ and $N$.

The following is one of the main technical results of the paper.

LEMMA 3.1. (a) The projection

$$
p: \mathscr{L}(M, N ; X) \rightarrow L(M, N ; X),
$$

given by sending a pair $(\nu, \varepsilon)$ to $\nu$, is a weak G-homotopy equivalence.

(b) Similarly, the natural projection

$$
p: \mathscr{C}(M, N ; X) \rightarrow C(M, N ; X)
$$

is a weak G-homotopy equivalence.

Lemma 3.1 is proved in $\S 6$. We now construct $G$-maps

$$
\begin{aligned}
& \alpha: \mathscr{L}(M, N ; X) \rightarrow \Gamma(M, N ; X) \text { and } \\
& \alpha: \mathscr{C}(M, N ; X) \rightarrow \Gamma(M, N ; X)
\end{aligned}
$$

for an appropriate choice of $\varepsilon_{0}$.

Construction 3.2. We first give the construction for $\mathscr{L}$. Let $\nu$ be a PL-arc appearing in an element of $L(M, N ; X)(\varepsilon)$. If $\varepsilon \leq \varepsilon_{0}$ (which 
has yet to be specified), and if $d_{M^{\prime}}\left([\nu], \pi\left(N_{1}\right)\right) \geq \varepsilon$, we think of $\nu$ as representing an inclusion of the standard cylinder $C=D^{n} \times Y$ in $M$, where $y=\operatorname{cl}(\operatorname{supp}(\nu))$, with image the corresponding cylinder $\bar{C}(\nu, \varepsilon)$. Such an inclusion $l$ is specified as follows. First embed $D^{n}$ into $M^{\prime}$ via the exponential map $e$, so that the center of the disc maps to $[\nu]$ and the image has radius $\varepsilon$. On $Y$ we take $l$ to be the identity, and on a point $(d, y)$ we take the unique lift of the path $t \mapsto e(t d)$ in $M^{\prime}$ to a path in $M$ starting at $y$ and normal to the fibers. (Although there is a rotational ambiguity in the construction of $l$, our constructions below are invariant under rotation.) When $d_{M^{\prime}}\left([\nu], \pi\left(N_{1}\right)\right)<\varepsilon, \nu$ may be represented by inclusion of $C \cap(P \times Y)$ in $M$, where $P$ is the half-space above some affine hyperplane in $\mathbb{R}^{n}$. The choice of $\varepsilon_{0}$ is now determined by the requirement that the $\varepsilon$ cylinders are diffeomorphic images of the standard cylinder. We shall define the desired section $\alpha(\nu)$ by first specifying a non-zero section $\lambda$ of $\tau_{C^{\prime}}=\tau_{C} \oplus \mathbb{R}$. This amounts to specifying a map $D^{n} \times Y \rightarrow S^{n+1}$.

On $D^{n} \times[-1,1]$, we have two norms: the usual Euclidean norm $\|v\|_{2}$ and the norm $\|v\|^{\prime}=\max \left\{\left\|\left(v_{1}, \ldots, v_{n}\right)\right\|_{2},\left|v_{n+1}\right|\right\}$. Define $\lambda: D^{n} \times[-1,1] \rightarrow S^{n+1}$ by

$$
\lambda(v)=\left(\left(\sin \pi\|v\|^{\prime}\right) v /\|v\|_{2^{\prime}}-\cos \pi\|v\|^{\prime}\right) .
$$

The PL-map $\nu$ determines a partition $a_{0}, a_{1}, \ldots, a_{n}$ of $Y$ into intervals on which $\nu$ is linear and does not cross the basepoint $e$, and we define the section of $\tau_{C^{\prime}}$ piecewise on subintervals $\left[a_{i}, a_{i+1}\right]$ as follows. The restriction of $\nu$ to this subinterval determines an affine isomorphism $\theta_{i}:\left[a_{i}, a_{i+1}\right] \rightarrow\left[b_{i}, b_{i+1}\right] \subset[-1,1]$. The map $\theta_{i}^{-1}$ extends to a smooth embedding $\phi_{i}: D^{n} \times\left[b_{i}, b_{i+1}\right] \rightarrow M$ whose image is the cylinder $\bar{C}\left(\nu \mid\left[a_{i}, a_{i+1}\right], \varepsilon\right)$. The derivative of $\phi_{i}$ now maps the field $\lambda$ to a non-zero section of $\tau_{M} \oplus \mathbb{R}$ defined on $\bar{C}\left(\nu \mid\left[a_{i}, a_{i+1}\right], \varepsilon\right)$. Continuitng this process piecewise, we obtain a non-zero section of $\tau_{M} \oplus \mathbb{R}$ defined on $\bar{C}(\nu, \varepsilon)$. Finally, a section defined on all of $M$ is obtained by extending via the constant section.

By combining sections defined in this way, we obtain a continuous map

$$
\alpha(\varepsilon): L(M, N ; X)(\varepsilon) \rightarrow \Gamma(M, N ; X)
$$

for each $\varepsilon \leq \varepsilon_{0}$, and these together determine the map $\alpha$.

Turning to the configuration space models, we could do a similar construction, or we can take the following shortcut: Regard $\mathscr{C}(M, N ; X)$ as a subspace of $\mathscr{L}(M \times I, N \times I ; X)$ by identifying a point $p$ in $M$ with the element $[p, \nu] \in \mathscr{L}(M \times I, N \times I, X)$, where 
$\nu: I \rightarrow S^{1}$ is the quotient $I \rightarrow S^{1}=[-1,1] / \approx$. By the construction of $\alpha$, its restriction to $M \times\{0\}$ takes values in $\Gamma(M, N ; X) \subset$ $\Gamma(M \times I, N \times I ; X)$, and we take as the approximation map the restriction, $\alpha \mid: \mathscr{C}(M, N ; X) \rightarrow \Gamma(M, N ; X)$. It is a straightforward task to check that this map agrees, up to homotopy, with the approximation map of Hauschild [H].

One also has a natural map $\delta: \mathscr{C}(M, N ; X) \rightarrow \mathscr{L}(M, N ; X)$ defined as the union of maps $\delta(\varepsilon): C(M, N ; X)(\varepsilon) \rightarrow L(M, N ; X)(\varepsilon)$; $\delta(\varepsilon)$ takes a point in $M$ to the PL-arc of degree 1 and length $\varepsilon$ centered at that point. Further, one can check that $\alpha \circ \delta \simeq \alpha$ as $G$-maps.

In view of Lemma 3.1, Theorem 1.2 now reduces to the following.

Proposition 3.3. (a) $\alpha: \mathscr{L}(M, N ; X) \rightarrow \Gamma(M, N ; X)$ is a weak homotopy equivalence.

(b) If each $X^{H}$ is connected and nonempty, then $\alpha: \mathscr{C}(M, N ; X) \rightarrow$ $\Gamma(M, N ; X)$ is weak homotopy equivalence.

Proposition 3.3 will be proved in $\S 5$, once we develop the necessary inductive machinery in $\S 4$.

4. Inductive steps. Our proof of Proposition 3.3 will be an induction on the dimension of $M$. At each stage of the induction, we shall proceed by breaking up our manifolds into pieces in several ways.

Lemma 4.1 will be used in regarding $M$ as decomposed in such a way as to cover an equivariant handlebody decomposition of $M^{\prime}$. (This is analogous to the main inductive step in Hauschild's work [H].) Lemma 4.2 is then used, in the case of circular fibers, to decompose simple manifolds of the form $G \times{ }_{H}\left(D(V) \times S^{1}\right)$ into copies of $G \times{ }_{H} D(V) \times I$, where $G$ acts trivially on $I$. Next, Lemmas 4.3 and 4.4 are used either to reduce arguments about the relative versions of the approximations to the corresponding absolute versions or to reduce to the case of lower dimensional manifolds. The first of these two lemmas refers to parts of the boundary consisting of whole fibers, while the second to parts of the boundary on the ends of fibers. Finally, Lemma 4.8 is used in an orbit-type induction to reduce to the free case.

First then, suppose that $M_{1}^{\prime}$ and $M_{2}^{\prime}$ are $G$-invariant codimensionzero submanifolds of $M^{\prime}$, with $M^{\prime}=M_{1}^{\prime} \cup M_{2}^{\prime}$, joined along a codimension-zero submanifold $B^{\prime}=\partial M_{1}^{\prime} \cap \partial M_{2}^{\prime}$ of their boundary. Let $M_{i}=\pi^{-1}\left(M_{i}^{\prime}\right)$ and $B=\pi^{-1}\left(B^{\prime}\right)$, so that $M=M_{1} \cup M_{2}$ and $B=$ $\partial M_{1} \cap \partial M_{2}$. 
If $N$ is a codimension-zero submanifold of $\partial M$, then restriction to $M_{1}$ determines maps

$$
\begin{gathered}
\rho: L(M, N ; X) \rightarrow L\left(M_{1},\left(M_{1} \cap N\right) \cup B ; X\right), \\
r: \mathscr{L}(M, N ; X) \rightarrow \mathscr{L}\left(M_{1},\left(M_{1} \cap N\right) \cup B ; X\right),
\end{gathered}
$$

and

$$
R: \Gamma(M, N ; X) \rightarrow \Gamma\left(M_{1},\left(M_{1} \cap N\right) \cup B ; X\right) .
$$

When discussing the analogous configuration space models, we take $M=M_{1} \cup M_{2}$, joined along a codimension-zero submanifold $B=$ $\partial M_{1} \cap \partial M_{2}$ of their boundary, and obtain similar restriction maps

$$
\rho: C(M, N ; X) \rightarrow C\left(M_{1},\left(M_{1} \cap N\right) \cup B ; X\right),
$$

and

$$
r: \mathscr{C}(M, N ; X) \rightarrow \mathscr{C}\left(M_{1},\left(M_{1} \cap N\right) \cup B ; X\right) .
$$

Recall [H] that a $G$-quasifibration is a $G$-map $p: E \rightarrow B$ such that $p^{K}: E^{K} \rightarrow B^{K}$ is a quasifibration for each $K \subset G$.

LEMMA 4.1. (a) With $C=\operatorname{cl}\left(\left(M_{2} \cap N\right)-B\right)$, the maps $\rho$ are $G$ quasifibrations with fibers $F=L\left(M_{2}, C ; X\right)$ and $C\left(M_{2}, C ; X\right)$ respectively.

(b) The sequences

$$
\mathscr{L}\left(M_{2}, C ; X\right) \stackrel{i}{\rightarrow} \mathscr{L}(M, N ; X) \stackrel{r}{\rightarrow} \mathscr{L}\left(M_{1},\left(M_{1} \cap N\right) \cup B ; X\right)
$$

and

$$
\mathscr{C}\left(M_{2}, C ; X\right) \stackrel{i}{\rightarrow} \mathscr{C}(M, N ; X) \stackrel{r}{\longrightarrow} \mathscr{C}\left(M_{1},\left(M_{1} \cap N\right) \cup B ; X\right),
$$

with $i$ the evident inclusions, are weak G-fibration sequences. (That $i s$, the inclusions $i$ induce weak equivalences of their sources into the homotopy-theoretic fibers of the maps $r$.)

(c) The map $R$ is a fibration, with each fiber equivalent to $\Gamma\left(M_{2}, C ; X\right)$. Moreover, the diagram

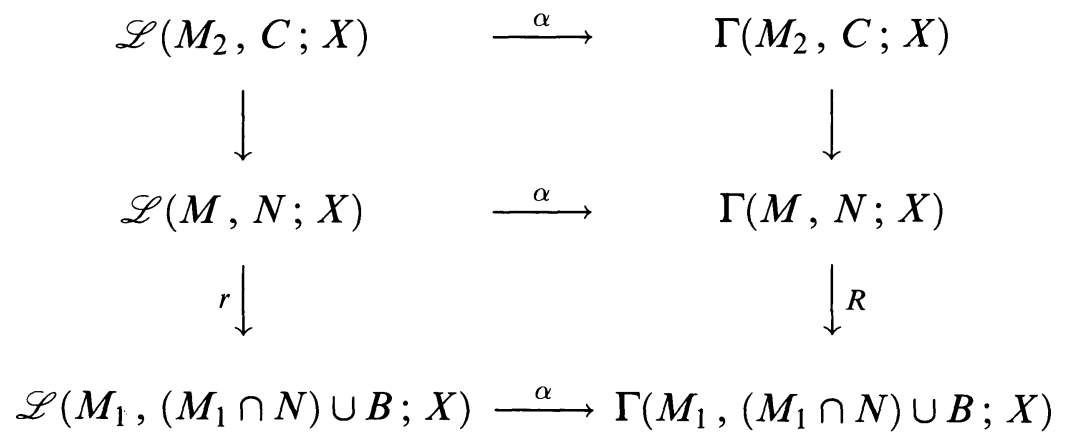

commutes, as does the similar diagram with the $\mathscr{L}$ 's replaced by $\mathscr{C}$ 's. 
The proofs of Lemmas 4.1-4.4 are deferred to $\S 6$.

We now consider a situation similar to the one above, but where we decompose $M$ across fibers in a nice way. Specifically, let $G$ act on $S^{1}$ via some group homomorphism $G \rightarrow S^{1}$, and let $M=M^{\prime} \times S^{1}$ with the product $G$-action, where $M^{\prime}$ is any $G$-manifold; let $\pi: M \rightarrow M^{\prime}$ be the projection. Let $S^{1}=(G / H \times I) \cup_{\partial}(G / H \times I)$ as a $G$-space, where $G / H$ is cyclic, and let $M_{1} \cong M_{2} \cong M^{\prime} \times(G / H \times I)$ be the corresponding submanifolds of $M$, so that $M=M_{1} \cup M_{2}$. As before, let $B=\partial M_{1} \cap \partial M_{1}$ and let $N$ be a codimension-zero submanifold of $\partial M$. One again has restriction maps

$$
\begin{aligned}
& \rho: L(M, N ; X) \rightarrow L\left(M_{1},\left(M_{1} \cap N\right) \cup B ; X\right), \\
& r: \mathscr{L}(M, N ; X) \rightarrow \mathscr{L}\left(M_{1},\left(M_{1} \cap N\right) \cup B ; X\right),
\end{aligned}
$$

and

$$
R: \Gamma(M, N ; X) \rightarrow\left(M_{1},\left(M_{1} \cap N\right) \cup B ; X\right)
$$

LEMMA 4.2. With $M_{1}$ and $M_{2}$ as above, and ignoring the configuration space models, the conclusions (a), (b) and (c) of Lemma 4.1 are true.

We next consider $G$-manifolds of the form $M=D V \times I$ with $N=N^{\prime} \times I$, where $N^{\prime}$ is a codimension-zero submanifold of $S V$. In this case the restriction we consider takes the form

$$
\rho: \mathscr{L}(M, N ; X) \rightarrow L(N ; \Sigma X) .
$$

$\rho$ is defined by

$$
\rho\left[\nu_{1}, \ldots, \nu_{n} ; x_{1}, \ldots, x_{n} ; \varepsilon\right]=\left[\xi_{1}, \ldots, \xi_{n} ;\left[x_{1}, t_{1}\right], \ldots,\left[x_{n}, t_{n}\right]\right],
$$

where $t_{i}=0$ unless $\nu_{i}$ is within $\varepsilon$ of $N$, in which case $\xi_{i}$ is the radial projection of $\nu_{i}$ into $N$, and $t_{j}=d\left(\nu_{i}, N\right) / \varepsilon$ (here the suspensions coordinates run from 0 to 1$)$. We can also describe a map

$$
r: \mathscr{L}(M, N ; X) \rightarrow \mathscr{L}(N ; \Sigma X)
$$

by essentially the same formula

$$
\begin{aligned}
& r\left[\nu_{1}, \ldots, \nu_{n} ; x_{1}, \ldots, x_{n} ; \varepsilon\right] \\
& \quad=\left[\xi_{1}, \ldots, \xi_{n} ;\left[x_{1}, t_{1}\right], \ldots,\left[x_{n}, t_{n}\right] ; \varepsilon / 2\right] .
\end{aligned}
$$

Dividing $\varepsilon$ by 2 insures, by elementary geometry, that the resulting cylinders do not overlap. 
Finally, there is a restriction

$$
R: \Gamma(M, N ; X) \rightarrow \Gamma(N ; \Sigma X) .
$$

For this, notice that $\tau_{M} \mid N=\tau_{N} \oplus \mathbb{R}$, so $F\left(\tau_{M} \mid N\right) \cong F\left(\tau_{N}\right) \wedge \mathscr{F} S^{1}$ and $F\left(\tau_{M} \mid N\right) \wedge_{F} X \cong F\left(\tau_{N}\right) \wedge_{\mathscr{F}} \Sigma X . R$ is simply given by restriction to $N$.

LemMA 4.3. (a) With $M=D V \times I$ and $N$ as above, the map

$$
\rho: \mathscr{L}(M, N ; X) \rightarrow L(N ; \Sigma X)
$$

is a G-quasifibration with fiber $F=\mathscr{L}(M ; X)$. With $M=D V$, and $N$ a codimension-zero submanifold of $S V$, the corresponding statement is true with $\mathscr{L}$ replaced by $\mathscr{C}$ and $L$ replaced by $C$.

(b) The sequence

$$
\mathscr{L}(M ; X) \stackrel{i}{\rightarrow} \mathscr{L}(M, N ; X) \stackrel{r}{\longrightarrow} \mathscr{L}(N ; \Sigma X)
$$

is a weak G-fibration sequence, as is the corresponding sequence for $\mathscr{C}$.

(c) The analogous map for $\Gamma$ is a fibration, and the map $\alpha$ induces a map of fiber sequences, as in the previous lemmas.

For the next lemma, we again consider $M=D V \times I$, but now let $N=D V \times\{-1\} \cup N^{\prime} \times I \cup N^{\prime \prime}$ where $N^{\prime} \subset S V$ and $N^{\prime \prime} \subset D V \times 1$ are codimension-zero submanifolds. We have a restriction

$$
\rho: L(M, N ; X) \rightarrow C\left(D V, N^{\prime} ; \Sigma X\right)
$$

given by

$$
\begin{aligned}
& \rho\left[\nu_{1}, \ldots, \nu_{n} ; x_{1}, \ldots, x_{n}\right] \\
& \quad=\left[\left[\nu_{1}\right], \ldots,\left[\nu_{n}\right] ;\left[x_{1}, \nu_{1}(-1)\right], \ldots,\left[x_{n}, \nu_{n}(-1)\right]\right]
\end{aligned}
$$

where it is now more convenient to think of the suspension coordinates as running from -1 to 1 . We can define a map $\mathscr{L}(M, N ; X) \rightarrow$ $\mathscr{C}\left(D V, N^{\prime} ; \Sigma X\right)$ in the same way, except that we also remember $\varepsilon$. Finally, there is an analog for the spaces of sections, defined as before Lemma 4.3.

LEMMA 4.4. With $M=D V \times I$ and $N$ as above, the map

$$
\rho: L(M, N ; X) \rightarrow C\left(D V, N^{\prime} ; \Sigma X\right)
$$


is a G-quasifibration with fiber $F=L\left(M, N^{\prime} \times I \cup N^{\prime \prime} ; X\right)$. If we replace $L$ with $\mathscr{L}$ and $C$ with $\mathscr{C}$ we obtain a weak $G$-fibration sequence. The corresponding sequence for $\Gamma$ is a G-fibration, and $\alpha$ induces a map of $G$-fiber sequences.

The next inductive sequence is essentially a variant of the main inductive sequence in [CW2]. As in the proof of Theorem 2.1, if $K \subset G$ we denote the $K$-fixed subspace of $L(M, N ; X)$ by $L_{K}(M, N ; X)$, and similarly for $\mathscr{L}(M, N ; X)$. If $\mathscr{F}$ is a collection of subgroups of $G$ closed under conjugacy, define subspaces $L_{G}(M, N ; X)_{\mathscr{F}} \subset$ $L_{G}(M, N ; X)$ and $\mathscr{L}_{G}(M, N ; X)_{\mathscr{F}} \subset \mathscr{L}_{G}(M, N ; X)$ by considering only PL-arcs of orbit type in $\mathscr{F}$, i.e., the $\nu_{i}$ appearing as factors have $G_{\left[\nu_{l}\right]} \in \mathscr{F}$. As in Theorem 2.1, we have

$$
L_{G}(M, N ; X)_{\mathscr{F}} \cong \prod_{\langle H\rangle \subset \mathscr{F}} L_{G}(M, N ; X)_{(H)}
$$

In particular, if $\mathscr{F}_{1} \subset \mathscr{F}_{2}$ are two families (that is, collections closed under subconjugacy), with $\mathscr{F}_{2}-\mathscr{F}_{1}$ consisting of the single conjugacy class $(H)$, then we have a product bundle sequence

$$
L_{G}(M, N ; X)_{\mathscr{F}_{1}} \stackrel{i}{\rightarrow} L_{G}(M, N ; X)_{\mathscr{F}_{2}} \stackrel{p}{\rightarrow} L_{G}(M, N ; X)_{(H)} .
$$

Note further that $L_{G}(M, N ; X)_{(H)}$ is homeomorphic to

$$
L_{W H}\left(M_{H}, N_{H} ; X^{H}\right) \text {, }
$$

where $Y_{H}$ denotes $Y^{H}-\bigcup_{K \supsetneqq H} Y^{K}$. Recall that, if $W$ is a regular neighorhood of $\bigcup_{K \supsetneqq H} Y^{K}$, we denote $M^{H}-W$ by $\widetilde{M}_{H}$, and that $W H$ acts freely on $M_{H}$ and $\widetilde{M}_{H}$. There is a sequence analogous to (4.5) using $\mathscr{L}$ instead of $L$ :

$$
\mathscr{L}_{G}(M, N ; X)_{\mathscr{F}_{1}} \stackrel{i}{\rightarrow} \mathscr{L}_{G}(M, N ; X)_{\mathscr{F}_{2}} \stackrel{p}{\longrightarrow} \mathscr{L}_{G}(M, N ; X)_{(H)} .
$$

In a similar vein, let $\Gamma_{G}(M, N ; X)_{\mathscr{F}}$ denote the space of sections $\sigma \in$ $\Gamma_{G}(M, N ; X)$ (the space of $G$-invariant sections) such that $\sigma(m)=*$ unless $m$ has orbit type in $\mathscr{F}$. We have a sequence

$$
\begin{aligned}
\Gamma_{G}(M, N ; X)_{\mathscr{F}} & \stackrel{l}{\rightarrow} \Gamma_{G}(M, N ; X)_{\mathscr{F}} \\
& \stackrel{\pi}{\rightarrow} \Gamma_{N H}\left(M^{H}, N^{H} ; X^{H}\right)_{(H)},
\end{aligned}
$$

where $\pi$ is given by restriction to fixed sets. It is not difficult to see that the space $\Gamma_{N H}\left(M^{H}, N^{H} ; X^{H}\right)_{(H)}$ is $G$-homotopy equivalent to $\Gamma_{W H}\left(\widetilde{M}_{H}, \widetilde{N}_{H} ; X^{H}\right)$ via the map induced by inclusion. Further, the simple argument in [CW2; Theorem 2.4] applies to show the following 
LEMMA 4.8. The sequence (4.6) is equivalent to (4.5), and the sequence (4.7) is a fiber sequence. Further, the maps $\alpha$ take the sequence (4.6) into the sequence (4.7) via a homotopy commutative diagram of maps. The corresponding statements are also true when $\mathscr{L}$ and $L$ are replaced by $\mathscr{C}$ and $C$.

Proof. The only part that needs comment is the fact that $\pi$ maps onto $\Gamma_{N H}\left(M^{H}, N^{H} ; X^{H}\right)_{(H)}$; this is true for dimensional reasons, as in previous lemmas.

5. Proof of Proposition 3.3. We first prove the case for $\mathscr{C}$ (part (b)), and then use this to prove the case for $\mathscr{L}$ (part (a)). Both steps will proceed by induction on the dimension $n$ of $M$.

Part (b). The map $\alpha: \mathscr{C}(M, N ; X) \rightarrow \Gamma(M, N ; X)$ is a G-equivalence if each $X^{H}$ is connected.

The start of the induction is the case $n=0$ which is trivial. The inductive step proceeds by a consideration of various cases. Let $n \geq$ 1 .

Case 1. $M=G \times D^{n}$, where $G$ acts trivially on the disc $D^{n}, N=\varnothing$.

This easily reduces to the nonequivariant case. Indeed, if $H \subset G$, then $\mathscr{C}_{H}(M ; X) \cong \prod_{H \backslash G} \mathscr{C}\left(D^{n} ; X\right)$, and similarly for the corresponding space of sections, with the map $\alpha$ respecting the product structures. The result follows from the nonequivariant case, with $M=D^{n}$, proved in [M1], since the map $\alpha$ and the approximating map described by May are homotopic.

Alternatively, let $K$ be a disc in the boundary of $D^{n}$. Then we can use the following sequence from Lemma 4.3:

$$
\mathscr{C}(M ; X) \rightarrow \mathscr{C}(M, G \times K ; X) \rightarrow \mathscr{C}(G \times K ; \Sigma X) .
$$

Applying $\alpha$ to compare this with the correspondence sequence of sections, we see that the total spaces are contractible and the base spaces are equivalent by induction, so the fibers are equivalent.

Case $1^{\prime} . M=G \times D^{n}, N$ arbitrary.

This follows from the fibration sequence of Lemma 4.3:

$$
\mathscr{C}(M ; X) \rightarrow \mathscr{C}(M, N ; X) \rightarrow \mathscr{C}(N ; \Sigma X) .
$$

$\alpha$ is an equivalence on the fibers by Case 1 , and on the base spaces by induction.

Case 2. $M$ a free $G$-manifold of dimension $n, N$ arbitrary. 
The result here is obtain by using a $G$-handlebody decomposition of $M$. The handlebodies will be of the form $G \times D^{n}$, and the reduction to the case of a single $G$-handle proceeds by induction on the number of handles using Lemma 4.1. For the case of a single $G$-handlebody, we appeal to Case $1^{\prime}$.

Case 3. $\operatorname{dim} V=n, M=D V, N$ arbitrary.

Here, we use Lemma 4.8 and induction on families, starting with the family $\{e\}$ and successively adding conjugacy classes of minimal subgroups not yet in the family. At each step the approximating map is a weak equivalence on base spaces by Case 2 .

Case 4. $M$ and $N$ arbitrary.

Take a $G$-handlebody decomposition of $M$, where the resulting handles have the form $G \times_{H} D V$. Lemma 4.1 and induction on the number of handles reduces us to proving that $\alpha$ is an equivalence on these handles, and this follows from Case 3.

Part (a). The map $\alpha: \mathscr{L}(M, N ; X) \rightarrow \Gamma(M, N ; X)$ is a G-equivalence.

The start of the induction, $n=1$, requires some argument. If $M=G / H \times I$ and $N=\varnothing$, then we use the sequence of Lemma 4.4:

$$
\mathscr{L}(M ; X) \rightarrow \mathscr{L}(M, G / H \times 0 ; X) \rightarrow \mathscr{C}(G / H ; \Sigma X) .
$$

Comparing this to the corresponding sequence of sections, we see that the total spaces are contractible and the base spaces are equivalent, hence the fibers are equivalent. If instead $N=G / H \times\{0,1\}$, then we use the sequence

$$
\mathscr{L}(M, G / H \times 0 ; X) \rightarrow \mathscr{L}(M, N ; X) \rightarrow \mathscr{C}(G / H ; \Sigma X) .
$$

The other possibilities for $N$ are trivial. The remaining case is $M=$ $G \times_{H} S^{1}$, where $H$ acts by rotations on $S^{1}$ (recall that the action of $G$ must preserve the chosen direction along $S^{1}$ ). In this case, examination of the possible fixed-point sets shows that we can reduce to considering $G$ acting on $S^{1}$, and then we use Lemma 4.2 to reduce to the cases above.

We now consider various cases as in the proof of part (b). Let $n>1$.

Case 1. $M=G \times D^{n-1} \times I, N=\varnothing$.

As in part (b), this is easily reduced to the nonequivariant case with $M=D^{n-1} \times I$, and is then a variant of the result in [CW1]. To 
deduce it in this case, we use Lemma 4.4, with $M=D^{n-1} \times I$, and $N=D^{n-1} \times\{0\}$. In the resulting diagram of quasifibrations the map on base spaces is a weak equivalence by part (b), and both total spaces are contractible, so the map on fibers is a weak equivalence.

Case $1^{\prime} . M=G \times D^{n-1} \times I, N$ arbitrary.

Use Lemma 4.4 twice if necessary, and part (b), to reduce to the case $N=N^{\prime} \times I$. Then use Lemma 4.3 and induction to reduce to Case 1.

Case 2. $M=M^{\prime} \times I$, with $M^{\prime}$ a free $G$-manifold of dimension $n-1, N$ arbitrary.

The result here is obtained by using a handlebody decomposition of $M^{\prime}$; lifting such a decomposition to one of $M$ gives $G$-handles of the form $G \times D^{n-1} \times I$. The reduction to the case of a single $G$-handle proceeds by induction using Lemma 4.1 as in part (b), and for the case of a single $G$-handlebody, we appeal to Case $1^{\prime}$.

Case. 3. $\operatorname{dim} V=n-1, M=D V \times I, N$ arbitrary.

As in Case 3 of part (b), we use Lemma 4.8 and induction on families. At each step the approximating map is again a weak equivalence on base spaces by Case 2 .

Case. 4. $M$ and $N$ arbitrary.

Take a $G$-handlebody decomposition of $M^{\prime}$, then lift to a decomposition of $M$. The resulting handles have the form $G \times_{H} D V \times Y$. Lemma 4.1 and induction on the number of handles reduces us to proving that $\alpha$ is an equivalence on these handles. We can use Lemma 4.2 to further reduce to the case where $Y=I$, and this follows from Case 3.

6. Proofs of some lemmas. Here we supply the technical proofs of Lemmas 3.1, and 4.1-4.4. All proofs in this section will use the Dold-Thom criterion [DT] for quasifibrations, in the form given by May [M1], which is this. Let $p: E \rightarrow B$ be a $G$-map which we wish to show is a quasifibration. If $K \subset G$, call a subspace $V$ of $B^{K}$ distinguished if the restriction $p^{K} \mid\left(p^{K}\right)^{-1}(V)$ is a quasifibration. The criterion implies that $p^{K}$ is a quasifibration if one is given a filtration $\left\{U_{i}\right\}$ of $B^{K}$ such that:

(i) every open subset of $U_{i}-U_{i-1}$ is distinguished. 
(ii) there is a deformation $h$ of a neighborhood $U$ of $U_{i-1}$ in $U_{i}$ and a covering homotopy $H:\left(p^{K}\right)^{-1}(U) \rightarrow\left(p^{K}\right)^{-1}(U)$ such that:

(1) $h_{0}$ is the identity and $h_{1}(U) \subset U_{i-1}$;

(2) $H_{0}$ is the identity and for all $t, p^{K} H_{t}=h_{t} p^{K}$;

(3) for all $z \in U$, the map $H_{1}:\left(p^{K}\right)^{-1}(z) \rightarrow\left(p^{K}\right)^{-1}\left(h_{1}(z)\right)$ is a homotopy equivalence.

In applying the criterion, we shall supply invariant filtrations and equivariant deformations which will restrict to the above data on fixed sets.

Before proving Lemma 3.1, we describe deformations of the space $\operatorname{PL}\left(Y, S^{1}\right)$ of PL-maps from a fiber $Y$ of $\pi: M \rightarrow M^{\prime}$ to $S^{1}$. Let $\rho \in[0,1)$, and let $\Delta_{\rho}: S^{1} \rightarrow S^{1}$ be the piecewise linear map induced by the map

$$
t \mapsto \begin{cases}-1 & \text { if } t \leq-1+\rho \\ t /(1-\rho) & \text { if }-1+\rho \leq t \leq 1-\rho ; \\ +1 & \text { if } t \geq 1-\rho,\end{cases}
$$

on $I$. Composition with $\Delta_{\rho}$ defines a deformation $d_{\rho}$ on the space $\operatorname{PL}\left(Y, S^{1}\right)$ preserving closure. Note that if $f \in \operatorname{PL}\left(Y, S^{1}\right)$ has the property that $f(Y) \subset(1-\rho, 1] \cup[-1,-1+\rho)$, then $d_{\rho}$ maps $f$ into the basepoint.

Proof of Lemma 3.1. For part (a), we consider the projection $p: \mathscr{L}(M, N ; X) \rightarrow L(M, N ; X)$. To prove the result it suffices to show that $p$ is a quasifibration with contractible fibers. That the fibers of $p$ are contractible is clear. The hard work comes in showing the quasifibration property. Roughly, we filter the base space by counting the number of fibers $Y$ on which PL-arcs are defined. On the complement of one filtration in the next, it is clear that $p$ restricts to a quasifibration. On the other hand, near points in the lower filtration, we show that it is possible to lower filtration through a deformation using parallel transport of arcs and deformations to degenerate points.

Precisely, the invariant filtration $\left\{U_{i}\right\}$ of $L(M, N ; X)$ we use in applying the Dold-Thom criterion is given by taking an element $\left[\nu_{1}, \ldots, \nu_{n} ; x_{1}, \ldots, x_{n}\right]$ to be in $U_{i}$ if the number of distinct points in $\left\{\left[\nu_{1}\right], \ldots,\left[\nu_{n}\right]\right\} \subset M^{\prime}$ is $\leq i$. If $V$ is an open subspace of $U_{i}$ $U_{i-1}$, define $\sigma: V \rightarrow \mathbb{R}$ by

$$
\begin{array}{r}
\sigma\left[\nu_{1}, \ldots, \nu_{n} ; x_{1}, \ldots, x_{n}\right]=\min \left\{\min _{\left[\nu_{1}\right] \neq\left[\nu_{j}\right]}\left\{d\left(\left[\nu_{i}\right],\left[\nu_{j}\right]\right)\right\} / 2,\right. \\
\left.\min _{i}\left\{d\left(\left[\nu_{i}\right], \pi(\partial M-N)\right)\right\}\right\}
\end{array}
$$


where the metric $d$ is taken in $M^{\prime}$. The map $\sigma$ is continuous and positive. We show that $V$ is distinguished by showing that $p \mid p^{-1}(V)$ is fiber homotopy equivalent to the identity fibration on $V$. Indeed, define $\psi: V \rightarrow p^{-1}(V)$ by

$$
\psi\left[\nu_{1}, \ldots, \nu_{n} ; x_{1}, \ldots, x_{n}\right]=\left(\left[\nu_{1}, \ldots, \nu_{n} ; x_{1}, \ldots, x_{n}\right], \varepsilon\right),
$$

where $\varepsilon=\min \left\{\sigma\left[\nu_{1}, \ldots, \nu_{n} ; x_{1}, \ldots, x_{n}\right], \varepsilon_{0}\right\}$. The composite $p \circ \psi$ is the identity, while $\psi \circ p$ is easily seen to be homotopic to the identity through a map over $V$, showing the claim.

For the second requirement of the Dold-Thom criterion we show that the pairs $\left(U_{i}, U_{i-1}\right)$ are equivariant NDR pairs, and that the associated homotopies lift to the total space level. With $C_{i}$ denoting $i$-fold configuration space, denote the subspace $\left(M^{\prime}\right)^{i}-C_{i}\left(M^{\prime}\right) \subset\left(M^{\prime}\right)^{i}$ by $\Delta$, so that $\Delta$ is a stratified $G \times \Sigma_{i}$-submanifold of $\left(M^{\prime}\right)^{i}$. It follows that there exists a $G \times \Sigma_{i}$-homotopy $J$ on $\left(M^{\prime}\right)^{i}$ with $J \mid \Delta$ the identity, $J_{0}$ the identity on $\left(M^{\prime}\right)^{i}$ and, for each $t \neq 0, J_{t}$ restricting to a retraction $W_{t} \rightarrow \Delta$ for some neighborhood $W_{t}$ of $\Delta$. Using parallel transport normal to the fibers we lift the homotopy $J$ to obtain an equivariant homotopy $\widetilde{J}$ on $\operatorname{Arc}(M)^{i}$ with $\widetilde{J}_{0}$ the identity and $\widetilde{J}$ constant over $\Delta$.

Since $X$ has a nondegenerate basepoint, there is a $G$-map $g: X \rightarrow$ $[0,1]$ with $g^{-1}(0)=*$. Let $f: \operatorname{Arc}(M)^{i} \subset X^{i} \rightarrow \mathbb{R}$ be given by

$$
f\left(\left(\nu_{j}\right),\left(x_{j}\right)\right)=\min \left\{\min _{j} \operatorname{diam}\left(\operatorname{Im} \nu_{j}\right), \min _{j} g\left(x_{j}\right)\right\} \text {. }
$$

With $L(M, N)(i) \subset \operatorname{Arc}(M)^{i}$ as above, $U_{i}$ is a quotient of $L(M, N)(i) \times X^{l}$. Define a $G$-map $R: L(M, N)(i) \times X^{i} \rightarrow \operatorname{Arc}(M)^{i} \times$ $X^{i}$ by

$$
R\left(\left(\nu_{j}\right),\left(x_{j}\right)\right)=\left(\widetilde{J}_{f\left(\left(\nu_{j}\right),\left(x_{j}\right)\right)}\left(\nu_{j}\right),\left(x_{j}\right)\right) .
$$

Recalling the map $d_{\rho}$ above, let $D_{\rho}: L(M, N)(i) \rightarrow L(M, N)(i)$ (or $\left.D_{\rho}: \operatorname{Arc}(M)^{i} \rightarrow \operatorname{Arc}(M)^{i}\right)$ be the map obtained by applying $d_{\rho}$ to each arc. Recalling the definition of $N_{1}$ before Theorem 1.2 , let $E^{\prime}: M^{\prime} \times$ $I \rightarrow M^{\prime}$ be a $G$-deformation which takes a neighborhood of $\pi\left(N_{1}\right)$ into $\pi\left(N_{1}\right)$. Then $E^{\prime}$ induces a corresponding deformation $E$ on $\operatorname{Arc}(M)$. Also, let $B: X \times I \rightarrow X$ be a based $G$-homotopy which contracts a neighborhood of the basepoint. Now take

$$
S: L(M, N)(i) \times X^{i} \rightarrow \operatorname{Arc}(M)^{i} \times X^{i}
$$

as the composite $R \circ\left(E_{1}^{i} \times 1\right) \circ\left(D_{1 / 2} \times B_{1}^{i}\right)$. Let $W_{j} \subset \operatorname{Arc}(M)^{i} \times$ $X^{i}$ consist of all points in $L(M, N)(i) \times X^{i}$ which map into $U_{j}$ under the identification map $v$. Then the restriction of $v \circ S$ to 
$S^{-1}\left(W_{i-1}\right)$ factors continuously and equivariantly through the quotient $Q=S^{-1}\left(W_{i-1}\right) / \approx \subset U_{i}$. We claim that $Q$ is an invariant neighborhood of $U_{i-1}$. Indeed, points $p=\left[\left(\nu_{j}\right),\left(x_{j}\right)\right]$ close to, but not in, $U_{i-1}$ possess one or more of the following features:

(a) One of the $\nu_{j}$ is close to the trivial PL-arc;

(b) One of the $x_{j}$ is close to the basepoint;

(c) One of the $\operatorname{supp} \nu_{i}$ is close to $N$;

(d) There exist $i$ and $j$ so that $\nu_{i}$ and $\nu_{j}$ are close to disjoint arcs on the same fiber. Thus in $R(p)$, the $i$ th and $j$ th arcs $\omega_{i}$ and $\omega_{j}$ will have supports that overlap only at points that they map close to the basepoint.

In all these cases, the map $\left(E_{1}^{i} \times 1\right) \circ\left(D_{1 / 2} \times B_{1}^{i}\right)$ brings $p$ into $R^{-1}\left(W_{i-1}\right)$, showing the assertion. A $G$-homotopy $h$ from the identity on $Q$ to $S: Q \rightarrow U_{i-1}$ is given for parameter values of $t$ in $[0,1 / 3]$ by homotoping the identity to $\left(D_{1 / 2} \times B_{1}^{i}\right)$ by means of the given homotopies on the factors, by $\left(E^{i} \times 1\right)$ on $[1 / 3,2 / 3]$, and for $t \in[2 / 3,1]$ by applying the homotopy $\widetilde{J}$.

We now describe a $G$-lift $H$ of the restriction of $h$ to a subspace of the form $V \times I$, where $V$ is an invariant open subspace of $Q$ containing $U_{i-1}$. First define $H^{\prime}: p^{-1}(Q) \times I \rightarrow L(M, N ; X) \times \mathbb{R}$ by the formula

$$
H^{\prime}((x, \varepsilon), t)= \begin{cases}(h(p(x), t), \varepsilon(1-3 t / 2)) & \text { if } t \leq 1 / 3 \\ (h(p(x), t), \varepsilon / 2) & \text { if } t \geq 1 / 3\end{cases}
$$

It remains to show that there exists a neighborhood $V$ as above with the restriction of $H^{\prime}$ to $V$ taking values in $\mathscr{L}(M, N ; X)$. Since $H^{\prime}$ covers the deformation $D_{t}:$ id $\simeq D_{1 / 2}$ during the first third of the homotopy, and $D_{t}$ does not decrease distances between supports, one can take $V=Q$ for this part. After this stage, distances between arcs may shrink to half their original values without leaving $\mathscr{L}(M, N ; X)$. Thus it is sufficient to observe that there is a neighborhood $V_{x}$ of each point $x \in U_{i-1}$ over which the deformation $H^{\prime}$ does not force cylinders of $1 / 2$ the original radius to overlap. Indeed we can choose $V_{x}$ so that arc supports are moved at most a distance that is small compared with the distances apart of arcs in $x$. Since in addition $H^{\prime}$ kills arcs of types (a), (b) and (c) above, there is no problem for pairs of arcs in an element of $V_{x}$ that do not merge to a single fiber under $H^{\prime}$. If, on the other hand, $\nu$ and $\mu$ are disjoint PL-arcs sufficiently close to a pair of disjoint arcs on the same fiber, then they will be carried under the second third of $h$ to a pair of arcs whose supports have disjoint closures under parallel transport 
and whose cylinders will not overlap at any stage of the homotopy. Since the fibers are contractible, these lifts are automatically fiberwise equivalences, showing the quasifibration property.

Turning to the proof of part (b) (for configuration space models), one filters the base space by counting the number of points in a configuration. Since there is no possibility of nearby points merging into a single point, there is no need for a deformation onto $\Delta$, and we can easily adapt the remaining arguments to this case. (A detailed proof in fact appears in [H].)

Proof of Lemma 4.1. We deal with the more difficult case of PLarc models, the case of configuration space models being similar but far simpler (and is in any case proved in [H]). In order to prove part (a) we again use the Dold-Thom criterion for quasifibrations, with the same filtration as in the proof of Lemma 3.1. Thus let $U_{k} \subset$ $L\left(M_{1},\left(M_{1} \cap N\right) \cup B ; X\right)$ consist of those collections of arcs involving at most $k$ fibers. If $V$ is an open subset of $U_{k}-U_{k-1}$, then with $F=L\left(M_{2}, C ; X\right)$, define a map

$$
t: V \times F \rightarrow \rho^{-1}(V)
$$

over $V$ by the formula

$$
\begin{aligned}
& t\left(\left[\mu_{1}, \ldots, \mu_{n} ; x_{1}, \ldots, x_{n}\right],\left[\nu_{1}, \ldots, \nu_{m} ; y_{1}, \ldots, y_{m}\right]\right) \\
& \quad=\left[\mu_{1}, \ldots, \mu_{n}, \nu_{1}, \ldots, \nu_{m} ; x_{1}, \ldots, x_{n}, y_{1}, \ldots, y_{m}\right],
\end{aligned}
$$

where none of the $\mu_{i}$ lie in $B$. We now claim that $t$ is an equivariant fiber homotopy equivalence, allowing us to conclude that $V$ is distinguished. To define a $G$-homotopy inverse $s: \rho^{-1}(V) \rightarrow V \times F$, first choose a collar of $B$ in $M_{2}$ diffeomorphic to $B \times[0,1)$, and use this to define a diffeomorphism $\varphi: M_{2}-B \times[0,1 / 2) \rightarrow M_{2}$ isotopic to the identity. If $\left[\mu_{1}, \ldots, \mu_{n}, \nu_{1}, \ldots, \nu_{m} ; x_{1}, \ldots, x_{n}, y_{1}, \ldots, y_{m}\right] \in$ $\rho^{-1}(V)$, with the arcs $\mu_{i}$ in $M_{1}-B$, and $\nu_{j}$ in $M_{2}$, let

$$
\begin{aligned}
& s\left[\mu_{1}, \ldots, \mu_{n}, \nu_{1}, \ldots, \nu_{m} ; x_{1}, \ldots, x_{n}, y_{1}, \ldots, y_{m}\right] \\
& \quad=\left(\left[\mu_{1}, \ldots, \mu_{n} ; x_{1}, \ldots, x_{n}\right],\left[\nu_{1} \varphi, \ldots, \nu_{m} \varphi ; y_{1}, \ldots, y_{m}\right]\right) .
\end{aligned}
$$

$s$ is continuous because $V \subset U_{k}-U_{k-1}$. It is easy to see that $s \circ t$ and $t \circ s$ are homotopic to the respective identities over $V$.

To show the neighborhood deformation property, the main point of the argument is that we can take PL-arcs in $M_{1}$ that are close to $B$, and deform them through $B$ and into $M_{2}$ on the total space 
level, following this below in the base space. We use this procedure to lower filtration, along with the expected deformations near degenerate elements.

Precisely, choose a bicollar $B \times[-1,1] \subset M$ with $B \times[-1,0] \subset$ $M_{1}$. Let $\Delta \subset\left(M_{1}^{\prime}\right)^{k}$ be the union of the complement of the $k$-fold configuration space and the space of $k$-tuples with at least one point in $B \times[-1,0]$. Since $\Delta$ is a stratified submanifold there exists a $G \times \Sigma_{k}$-homotopy $J$ on $\left(M_{1}^{\prime}\right)^{k}$ such that $J_{0}$ is the identity, $J \mid \Delta$ is constant, and for each $t \neq 0, J_{t}$ restricts to a retraction $W_{t} \rightarrow \Delta$ for some neighborhood $W_{t}$ of $\Delta$. Using parallel transport, lift $J$ to a $G \times \Sigma_{k}$-homotopy $\widetilde{J}$ on $\operatorname{Arc}\left(M_{1}\right)^{k}$, so that $\widetilde{J}_{0}$ is the identity, and $\widetilde{J}$ is constant over $\Delta$. Using this $\widetilde{J}$, we proceed as in the proof of Lemma 3.1 to define a map

$$
S: L\left(M_{1},\left(M_{1} \cap N\right) \cup B\right)(k) \times X^{k} \rightarrow \operatorname{Arc}\left(M_{1}\right)^{k} \times X^{k} .
$$

Further, choose an order preserving diffeomorphism $(-1,1) \rightarrow(-1,1)$ taking $(0,1 / 2)$ to $(-1 / 2,1 / 2)$. Using the bicollar $B \times(-1,1)$, this gives a diffeomorphism $\psi: M \rightarrow M$ isotopic to the identity. Restricting $\psi$ to $M_{1}$ gives a map $\psi^{*}: \operatorname{Arc}\left(M_{1}\right) \rightarrow \operatorname{Arc}\left(M_{1}\right)$. Finally, as in Lemma 3.1, we can see that $\left(\psi^{*}\right)^{k} \circ S$ induces a map $Q \rightarrow U_{k-1}$, where $Q \subset U_{k}$ is an invariant neighborhood of $U_{k-1}$, and a $G$-homotopy $h$ from the identity on $Q$ to this map, through maps into $U_{k}$.

$h$ lifts to $\rho^{-1}(Q)$ by an analogous construction: We extend $\widetilde{J}$ to $\operatorname{Arc}(M)^{k}$ by making it constant when at least one arc is in $M_{2}$, then extend to $\operatorname{Arc}(M)^{k} \times \operatorname{Arc}\left(M_{2}\right)^{l}, l \geq 0$, by making it the identity on the second factor. Using the full definition of $\psi$ then gives us the desired lift of $h$ to a homotopy $H$ on $\rho^{-1}(Q)$.

It remains to check that $H_{1}$ is a homotopy equivalence on each fiber. Fix a point $z=\left[\left(\nu_{l}\right),\left(x_{i}\right)\right]$ in $U_{k}$. The map $H_{1}: \rho^{-1}(z) \rightarrow$ $\rho^{-1}\left(h_{1}(z)\right)$ can be described as translation by a fixed configuration $\Lambda$ of arcs. Precisely, it is given by pushing a given collection of arcs in $M_{2}$ away from $B$ (using $\psi$ ) and introducing $\Lambda$ near $B$, these being the arcs from $z$ pushed across $B$ by $\psi$. A homotopy inverse to $H_{1}$ is specified by first pushing away from $B$ and then introducing the inverses of the arcs in $\Lambda$ near $B$ in the same position as those in $\Lambda$ itself. Using any choice of homotopies cancelling the arcs in $\Lambda$ with their inverses, it is easy to see that the map just described is a homotopy inverse to $H_{1}$. 
To show part (b) for the PL-arc case, consider the following diagram:

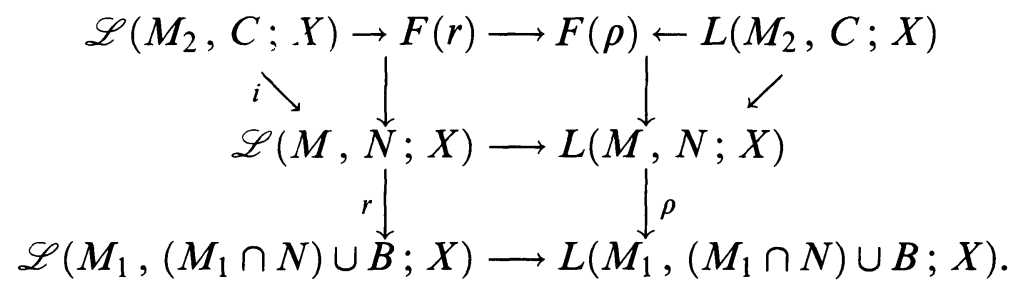

The spaces $F(r)$ and $F(\rho)$ are the homotopy fibers of $r$ and $\rho$. As a basepoint in $L\left(M_{1},\left(M_{1} \cap N\right) \cup B ; X\right)$ we use the empty collection of arcs; in $\mathscr{L}\left(M_{1},\left(M_{1} \cap N\right) \cup B ; X\right)$ we use the empty collection paired with some fixed $\varepsilon_{1}$. The middle two squares of the diagram obviously commute, and by Lemma 3.1 the three horizontal maps are all weak $G$-equivalences. To define the map $\mathscr{L}\left(M_{2}, C ; X\right) \rightarrow F(r)$, we need only specify a homotopy from $r \circ i$ to a map constant at the basepoint; since the image of $r \circ i$ is contained in the set $\left\{(\varnothing, \varepsilon) \mid \varepsilon \in\left(0, \varepsilon_{0}\right]\right\}$, we take a homotopy that simply contracts this set to the chosen basepoint in the obvious way. Writing down the maps explicitly, it is easy to see that the very topmost square of the diagram also commutes. Since, by part (a), the map $L\left(M_{2}, C ; X\right) \rightarrow F(\rho)$ is a weak $G$-equivalence, we can now conclude that $\mathscr{L}\left(M_{2}, C ; X\right) \rightarrow F(r)$ is also a weak $G$ equivalence.

Finally, to show part (c), it is easy to see that $R$ satisfies the homotopy-lifting property. Moreover, $R$ maps onto every component and the fibers over distinct components are equivalent to $\Gamma\left(M_{2}, C ; X\right)$, because, for dimensional reasons, every section over $B$ is homotopic to a trivial section. That the diagram in (c) commutes is obvious.

Proof of Lemma 4.2. Here, for the Dold-Thom criterion, we use a new filtration. Roughly speaking, this measures the total number of "zigs and zags" comprising the PL-arcs. Precisely, if $\nu$ is an indecomposable PL-arc, let $\lambda(\nu)$ be the number of linear segments in $\nu$ with non-trivial slope. Now let $U_{i} \subset L\left(M_{1},\left(M_{1} \cap N\right) \cup B ; X\right)$ consist of all $\left[\left(\nu_{j}\right) ;\left(x_{j}\right)\right]$ with each $\nu_{j}$ indecomposable and $\sum_{j} \lambda\left(\nu_{j}\right) \leq i$.

The idea of the proof that $\rho$ is a quasifibration is now this: In the complement of $U_{i-1}$ in $U_{i}$ we can show that $\rho$ is equivalent to a trivial fibration, by extending arcs defined only on $M_{1}$ to closed arcs defined on $M$, using the "addition of tails" construction detailed 
below to obtain closed arcs. The deformation of a neighborhood of $U_{i-1}$ in $U_{i}$ into $U_{i-1}$ is then constructed combining the ways in which arcs can deform in such a way as to lower the number of "zigs and zags," with the usual deformations to singularities. This can be followed in the total space level after providing enough extra "tail" to arcs in $M_{1}$ so that no nontrivial line segment need ever become horizontal.

Fix any $\delta \in(0,1 / 2)$ and let $M_{1}^{\prime}=M^{\prime} \times(G / H \times[-1-\delta, 1+\delta])$, considered as a submanifold of $M$ containing $M_{1}$. If $\nu$ is a PL-arc in $M_{1}$, we describe an extension $\tau(\nu)$ of $\nu$ to a closed arc in $M_{1}^{\prime}$ by means of the following "addition of tails" construction. Let $y \in Y$ be an endpoint of $Y$. If $\nu(y)=e$ we extend $\nu$ by means of the trivial tail in this direction. Otherwise, let $s$ be the linear segment of $\nu$ whose domain contains $y$, and let $s(y)=w$. If $s$ has slope $m$, extend $\nu$ by adding a segment of slope $\operatorname{sgn}(m) 2 / \delta$ beginning at $(y, w)$ and ending when it first hits the basepoint. $\tau$ is continuous in $U_{i}-U_{i-1}$.

With $V$ an open subset of $U_{i}-U_{i-1}$ and $F=L\left(M_{2}, C ; X\right)$, define

$$
r: V \times F \rightarrow \rho^{-1}(V)
$$

over $V$ by the formula

$$
\begin{array}{r}
r\left(\left[\mu_{1}, \ldots, \mu_{n} ; x_{1}, \ldots, x_{n}\right],\left[\nu_{1}, \ldots, \nu_{m}, y_{1}, \ldots, y_{m}\right]\right) \\
=\left[\tau\left(\mu_{1}\right), \ldots, \tau\left(\mu_{n}\right), \nu_{1} \circ \varphi, \ldots, \nu_{m} \circ \varphi ;\right. \\
\left.\quad x_{1}, \ldots, x_{n}, y_{1}, \ldots, y_{m}\right],
\end{array}
$$

where $\varphi$ maps each fiber $G / H \times I$ to itself by the formula

$$
(g H, t) \mapsto \begin{cases}(g H, t /(1-\delta)) & \text { if }|t| \leq 1-\delta ; \\ (g H,-1) & \text { if } t<-1+\delta ; \\ (g H,+1) & \text { if } t>1-\delta .\end{cases}
$$

To define a map $s: \rho^{-1}(V) \rightarrow V \times F$, we need another tail construction: Suppose given an $\operatorname{arc} \nu$ in $M$. Let $\nu_{1}$ and $\nu_{2}$ be the restrictions of $\nu$ to $M_{1}$ and $M_{2}$ respectively. Let $\sigma(\nu)$ be the closed arc in $M_{2}$ defined by composing $\nu_{2}$ with $\varphi$ above, and then adding tails on either end which are the opposites of the tails we would add to form $\tau\left(\nu_{1}\right)$. Precisely, these tails start at $e$ at the endpoints, are constant for a time, then proceed with slopes the negatives of those used in forming $\tau\left(\nu_{1}\right)$ until they connect with $\nu_{2} \circ \varphi . \sigma$ is then a continuous map $\rho^{-1}(V) \rightarrow F$. Combining with the projection $\rho$ gives $s$. Inspecting the composites $r \circ s$ and $s \circ r$, it is not hard to see that both are homotopy equivalences on fibers. 
Now we describe a deformation of a neighborhood of $U_{i-1}$ in $U_{i}$ into $U_{i-1}$. Let $L_{i}$ be the space of $(2 i+1)$-tuples of linear segments defined on subintervals of fibers of $M_{1}$. This is a $G \times \Sigma_{i}$-space. An element of this space is admissible if the supports of the segments are mutually disjoint, and they assemble together to give a collection $\left\{\nu_{i}\right\}$ of PL-arcs with $\sum_{j} \lambda\left(\nu_{j}\right) \leq i$. The subspace $A_{i}$ of admissible $i$-tuples is a $G \times \Sigma_{i}$-invariant subspace, and is a stratified $G \times \Sigma_{i}$-manifold. An element of $A_{i}$ is degenerate if $\sum_{j} \lambda\left(\nu_{j}\right)<i$; the subspace $D_{i}$ of the degenerate elements is a stratified $G \times \Sigma_{i}$-submanifold, and hence an equivariant strong neighborhood deformation retract of $A_{i}$. Let $K: W_{i} \times I \rightarrow W_{i}$ be a strong deformation of a neighborhood of $D_{i}$ into $D_{i}$, so that $K \mid W_{i} \times[0, t]$ is a strong deformation of a neighborhood of $D_{i}$ into $D_{i}$ for all $t$.

Now, $U_{i}$ is the surjective image of a certain subspace $A_{i}^{\prime} \subset A_{i} \times$ $X^{2 i+1}$, where there are restrictions on the allowed $X$-coordinates, and where $A_{i}^{\prime}$ is a $G \times \Sigma_{i}$-subspace.

As in Lemma 3.1, since $X$ has a nondegenerate basepoint, there is a continuous $G$-equivariant function $g: X \rightarrow[0,1]$ such $g(*)=0$ and $g(x) \neq 0$ if $x \neq *$. Let $f: A_{i} \times X^{2 i+1} \rightarrow \mathbb{R}$ be given by

$$
f\left(\left(\nu_{j}\right),\left(x_{j}\right)\right)=\left\{\begin{array}{l}
0 \text { if fewer than } i \nu_{j} \text { are nonconstant } \\
\min \left\{g\left(x_{j}\right): \nu_{j} \text { is nonconstant }\right\} \text { otherwise. }
\end{array}\right.
$$

Although this function is not continuous, the function

$$
R\left(\left(\left(\nu_{j}\right),\left(x_{j}\right)\right), t\right)=K_{t f\left(\left(\nu_{j}\right),\left(x_{j}\right)\right)}\left(\left(\nu_{j}\right),\left(x_{j}\right)\right)
$$

is continuous, because $K$ is constant on $D_{i}$.

Let $B$ and $E$ be as in the proof of Lemma 3.1. Let the homotopy

$$
S: A_{i}^{\prime} \times I \rightarrow A_{i} \times X^{2 i+1}
$$

be constant on $[0,1 / 3] ;\left(E^{2 i+1} \times 1\right) \circ\left(D_{s} \times\left(B_{2 s}\right)^{2 i+1}\right)$ on $[1 / 3,2 / 3]$, where $s=3(t-1 / 3) / 2$; and $R_{s} \circ\left(D_{1 / 2} \times\left(B_{1}\right)^{2 i+1}\right)$ on $[2 / 3,1]$, where $s=3(t-2 / 3)$.

Let $V_{j} \subset A_{i} \times X^{2 i+1}$ consist of all points in $A_{i}^{\prime}$ that map into $U_{j}$ under the identification map, which we call $v$. The restriction of $v \circ S$ to $S_{1}^{-1}\left(V_{i-1}\right)$ factors continuously and equivariantly through the quotient $Q=S_{1}^{-1}\left(V_{i-1}\right) / \approx \subset U_{i}$. We claim that there is an invariant neighborhood of $V_{i-1}$ contained in $S^{-1}\left(V_{i-1}\right)$ on which $S$ is a deformation taking values in $A_{i}^{\prime}$. In fact, the only thing that would take us out of $A_{i}^{\prime}$ is if two arcs on the same fiber indexed by two different points of $X$ were to be merged together by $R$. However, if we have a point in the lower filtration $V_{i-1}$, there is a neighborhood 
$T$ of the point in $A_{i}^{\prime}$ in which the slopes of all line segments are bounded. After the deformation $D_{s}$, we can conclude that there is a nonzero lower bound on the distance between arcs on the same fiber labeled with different points in $X$. Passing to a possibly smaller neighborhood, we can also assume that no arc is moved more than half this distance. Thus, the neighborhood $T$ will be carried into $A_{i}^{\prime}$ throughout the homotopy. The union of such neighborhoods over all the points of $V_{i-1}$ is the neighborhood of $V_{i-1}$ that we want. This in turn gives a neighborhood of $U_{i-1}$ in $U_{i}$ and a deformation $h$ of this neighborhood into $U_{i-1}$.

To lift this homotopy to a homotopy $H$ on a neighborhood of $\rho^{-1}\left(U_{i-1}\right) \subset \rho^{-1}\left(U_{i}\right) \subset L(M, N ; X)$ we do the following: Given $s \in S^{1}$, let $w_{s}$ be the arc on $[0, \delta]$ that starts at $s$, winds exactly once around $S^{1}$ in the positive direction, then winds around once the other way, to end at $s$. There is then a deformation $F$ of $L(M, N ; X)$ that contracts the part of any arc in $M_{2}$ using $\varphi$ above, and in the "gap" produced introduces the arc $w_{s}$, where $s$ is the value of the arc at $B$. During the course of this deformation we introduce $w_{s}$ in such a way that the slopes are never $0 . F$ is the first third of the deformation $H$, and clearly covers the first third of $h$. The remaining part of $H$ is given by deforming the parts of the arcs in $M_{1}$ by the homotopy $h$, and at the same time deforming the parts in $M_{2}$ using $E$. The arcs $w_{s}$ are modified by letting the endpoint in $B$ move according to $h$. Passing to a possibly smaller neighborhood of $U_{i-1}$, these endpoints will not wander far enough to force the large slope in $w_{s}$ to 0 . Thus $H$ is well-defined, and covers $h$. Further, on any fiber the action of $H_{1}$ is translation by a fixed collection of arcs, so is a homotopy equivalence.

The proofs of parts (b) and. (c) are identical to the proofs given in Lemma 4.1.

Proof of Lemma 4.3. Again we shall concentrate on the case of the PL-arc models, leaving the simpler configuration-space models. We may assume that the number $\varepsilon_{0}$ used in defining $\mathscr{L}$ is smaller than $1 / 2$. We use the same filtration as in the proof of Lemma 3.1; $U_{i}$ is the space of arcs involving $i$ or fewer fibers in $M$.

To show that $\rho$ is a quasifibration, we will do the following: In the complement of $U_{i-1}$ in $U_{i}$, it is easy to see that $\rho$ is equivalent to a trivial bundle by pushing arcs inwards from $N$. To construct the deformation of a neighborhood of $U_{i-1}$ into $U_{i-1}$, we use the same deformation that we used in proving Lemma 3.1. With a bit of care 
we see that we can follow this homotopy on the total space level by separating the arcs that are close to $N$ from those further away.

Let $V$ be an invariant open subspace of $U_{i}-U_{i-1}$ and define $\sigma: V \rightarrow \mathbb{R}$ as in Lemma 3.1. Choose an equivariant diffeomorphism $\gamma: M \rightarrow M \cup\left(S V \times I \times\left[0, \varepsilon_{0}\right]\right)$, where $S V \times I \times\left\{\varepsilon_{0}\right\}$ is identified with part of $\partial M$ in the obvious way. We can choose $\gamma$ so that $d(\gamma(x), \gamma(y)) \leq 2 d(x, y)$. If $\nu$ is an arc in $N$ and $0 \leq t \leq \varepsilon_{0}$, let $\widetilde{\gamma}(\nu, t)$ be the arc in $M$ given by considering the corresponding arc in $S V \times I \times\left[0, \varepsilon_{0}\right]$ on the fiber $([\nu], t)$, and then using $\gamma$ to consider this as an arc in $M$. Define $\psi: V \times F \rightarrow \rho^{-1}(V)$ by

$$
\begin{array}{r}
\psi\left(\left[\nu_{1}, \ldots, \nu_{n} ;\left[x_{1}, t_{1}\right], \ldots,\left[x_{n}, t_{n}\right]\right],\left[\mu_{1}, \ldots, \mu_{m} ; y_{1}, \ldots, y_{m}\right], \varepsilon\right) \\
=\left(\left[\tilde{\gamma}\left(\nu_{1}, t_{1} \varepsilon^{\prime}\right), \ldots, \tilde{\gamma}\left(\nu_{n}, t_{n} \varepsilon^{\prime}\right), \mu_{1} \gamma, \ldots, \mu_{m} \gamma\right.\right. \\
\left.\left.x_{1}, \ldots, x_{n}, y_{1}, \ldots, y_{m}\right], \varepsilon^{\prime}\right)
\end{array}
$$

where $\varepsilon^{\prime}=\min \left\{\sigma\left[\nu_{1}, \ldots, \nu_{n} ; x_{1}, \ldots, x_{n}\right] / 2, \varepsilon / 2\right\}$. A $G$-fiber homotopy inverse to $\psi$ is given by the product of projection to $V$ and the map $\rho^{-1}(V) \rightarrow F$ given by taking those arcs whose $\varepsilon$-cylinders do not meet $N$. This is continuous because $V \subset U_{i}-U_{i-1}$.

We take as a $G$-deformation $h: Q \times I \rightarrow Q$ of a neighborhood of $U_{i-1}$ in $U_{i}$ into $U_{i-1}$ the homotopy that is constant on $[0,1 / 2]$, and on $[1 / 2,1]$ is the homotopy constructed in the proof of Lemma 3.1, done at twice the speed. We describe a $G$-lift $H$ of the restriction of $h$ to a subspace of the form $V \times I$, where $V$ is an invariant open subspace of $Q$ containing $U_{i-1}$. First define $H^{\prime}: \rho^{-1}(Q) \times I \rightarrow$ $L(M, N ; X) \times \mathbb{R}$ as follows: A typical element of $\rho^{-1}(Q)$ can be written as $(x, y, \varepsilon)$, where $x$ is a configuration of arcs whose $\varepsilon$ cylinders meet $N$, and $y$ is a configuration of arcs whose $\varepsilon$-cylinders do not meet $N$. Let $\gamma_{t}$ be a homotopy from $\gamma_{0}=$ id to $\gamma_{1}=\gamma$. Then $H^{\prime}$ is given by

$$
H^{\prime}((x, y, \varepsilon), t)= \begin{cases}\left(x \gamma_{2 t}^{-1}, y \gamma_{2 t}^{-1}, \varepsilon(1-t)\right) & \text { if } t \leq 1 / 2 \\ \left(h\left(x \gamma^{-1}, t\right), y \gamma^{-1}, \varepsilon / 2\right) & \text { if } t \geq 1 / 2\end{cases}
$$

By the same reasoning as in the proof of Lemma 3.1, this gives the homotopy $H$ over a possibly smaller neighborhood of $U_{i-1}$. On any fiber $H$ is given by translation by a fixed configuration of arcs, so is a homotopy equivalence. 
Part (b) follows because the diagram

$$
\begin{gathered}
\mathscr{L}(M, N ; X) \\
r \swarrow \quad \searrow \rho \\
\mathscr{L}(N ; \Sigma X) \longrightarrow L(N, \Sigma X)
\end{gathered}
$$

commutes, which allows us to form a diagram analogous to the one we used in the proof of Lemma 4.1, so that we may use the same argument that we used there.

Finally, part (c) follows for the same reasons as in the previous lemmas.

Proof of Lemma 4.4. Start by filtering $C\left(D V, N^{\prime} ; \Sigma X\right)$ in the obvious way: $U_{i}$ consists of configurations with at most $i$ points. Choose a $\delta \in(0,1 / 2)$, and define $\varphi: I \rightarrow I$ by the formula

$$
\varphi(t)= \begin{cases}-1+2(t+1-\delta) /(2-\delta) & \text { if } t>-1+\delta \\ -1 & \text { if } t \leq-1+\delta\end{cases}
$$

Let $V \subset U_{i}-U_{i-1}$ be an open set and define

$$
r: V \times F \rightarrow \rho^{-1}(V)
$$

by

$$
\begin{array}{r}
r\left(\left[v_{1}, \ldots, v_{n} ;\left[x_{1}, t_{1}\right], \ldots,\left[x_{m}, t_{m}\right]\right],\left[\nu_{1}, \ldots, \nu_{n} ; y_{1}, \ldots, y_{n}\right]\right) \\
=\left[\tau\left(v_{1}, t_{1}\right), \ldots, \tau\left(v_{m}, t_{m}\right), \nu_{1} \varphi, \ldots, \nu_{n} \varphi ;\right. \\
\left.\quad x_{1}, \ldots, x_{m}, y_{1}, \ldots, y_{n}\right] .
\end{array}
$$

Here, $\tau(v, t)$ is the arc in $M$ defined on the fiber $v \times I$, and having

$$
\tau(v, t)(z)= \begin{cases}t+2(z+1) / \delta & \text { if } z<-1+\delta(1-t) / 2, \\ 1 & \text { otherwise. }\end{cases}
$$

We define $s: \rho^{-1}(V) \rightarrow V \times F$ as the product of projection to $V$ and the map $\sigma: \rho^{-1}(V) \rightarrow F$ defined by composing arcs with $\varphi$ and adding tails opposite to the tails $\tau$ above (cf. Lemma 4.2). It is now easy to see that $r \circ s$ and $s \circ r$ are homotopy equivalences on fibers.

We can deform a neighborhood of $U_{i-1}$ into $U_{i-1}$ by a homotopy $h$ that is constant on its first third, on its second third deforms a neighborhood of $N^{\prime}$ into $N^{\prime}$, and on its last third deforms a neighborhood of the basepoint in $\Sigma X$ to the basepoint. We can lift this to a deformation $H$ that in its first third uses $\varphi$ to deform the arcs, while adding the arcs $w_{s}$ used in Lemma 4.2; on its second part it 
deforms a neighborhood of $N^{\prime} \times I$ into $N^{\prime} \times I$, and on its last third follows the deformation $h$ on the -1 endpoints of the arcs, much as in Lemma 4.2. It is not hard to see that on fibers $H$ is a homotopy equivalence.

The last statements of the lemma follow in the usual way.

\section{REFERENCES}

[CW1] J. Caruso and S. Waner, An approximation to $\Omega^{n} \Sigma^{n} X$, Trans. Amer. Math. Soc., 265 (1) (1981), 147-162.

[CW2] _ An approximation theorem for equivariant loop spaces in the compact Lie case, Pacific J. Math., 117 (1) (1985), 27-49.

[CW3] S. R. Costenoble and S. Waner, Fixed set systems of equivariant infinite loop spaces, to appear, Trans. Amer. Math. Soc.

[D] T. tom Dieck, Transformation Groups and Representation Theory, Lect. Notes in Math., Springer-Verlag 766 (1979).

[DT] A. Dold and R. Thom, Quasifaserungen und unendlische symmetrische Produkte, Ann. of Math., (2) 67 (1958), 239-281.

[H] H. Hauschild, Aqquivariante Konfigurationsräume und Abbildungsräume, Proc. Topology Symp. Siegen. Lect. Notes Math., 788, Springer-Verlag (1980), 281-315.

[M1] J. P. May, The Geometry of Iterated Loop Spaces, Lect. Notes Math., 271, Springer-Verlag (1972).

[M2] D. McDuff, Configuration spaces of positive and negative particles, Topology, 14 (1975), 91-107.

[S] G. Segal, Configuration spaces and iterated loop spaces, Invent. Math., 21 (1973), 213-221.

[W] S. Waner, Equivariant homotopy theory and Milnor's Theorem, Trans. Amer. Math. Soc., 258 (2) (1980), 351-368.

[WW] S. Waner and Y. Wu, The local structure of tangent G-vector fields, Topology and Appl., 23 (1986), 129-143.

Received August 29, 1988 and in revised form January 25, 1989.

HOFSTRA UNIVERSITY

HEMPSTEAD, NY 11550, USA

AND

THE UNIVERSITY OF THE WITWATERSRAND

1 Jan SMUts AVENUE

JohanNesburg 2193, South Africa 



\section{PACIFIC JOURNAL OF MATHEMATICS EDITORS}

\author{
V. S. VARADARAJAN \\ (Managing Editor) \\ University of California \\ Los Angeles, CA 90024-1555-05 \\ Herbert Clemens \\ University of Utah \\ Salt Lake City, UT 84112 \\ Thomas ENRIGHT \\ University of California, San Diego \\ La Jolla, CA 92093
}

R. FINN

Stanford University

Stanford, CA 94305

Hermann FlaschKa

University of Arizona

Tucson, AZ 85721

VAUGHAN F. R. Jones

University of California

Berkeley, CA 94720

STEVEN KERCKHOFF

Stanford University

Stanford, CA 94305
C. C. MOORE

University of California

Berkeley, CA 94720

Martin SCharlemanN

University of California

Santa Barbara, CA 93106

HAROLd STARK

University of California, San Diego

La Jolla, CA 92093

\section{ASSOCIATE EDITORS}
R. ARENS
E. F. BECKENBACH
B. H. NeumanN
F. WoLF
(1904-1989)
K. YoshidA (1906-1982)

\section{SUP}

UNIVERSITY OF ARIZONA

UNIVERSITY OF BRITISH COLUMBIA

CALIFORNIA INSTITUTE OF TECHNOLOGY

UNIVERSITY OF CALIFORNIA

MONTANA STATE UNIVERSITY

UNIVERSITY OF NEVADA, RENO

NEW MEXICO STATE UNIVERSITY

OREGON STATE UNIVERSITY
UNIVERSITY OF OREGON

UNIVERSITY OF SOUTHERN CALIFORNIA

STANFORD UNIVERSITY

UNIVERSITY OF HAWAII

UNIVERSITY OF TOKYO

UNIVERSITY OF UTAH

WASHINGTON STATE UNIVERSITY

UNIVERSITY OF WASHINGTON 


\section{Pacific Journal of Mathematics}

Vol. 144, No. $1 \quad$ May, 1990

Wojciech Chojnacki, On some totally ergodic functions $\ldots \ldots \ldots \ldots \ldots \ldots 1$

Steven R. Costenoble, Stefan Waner and G. S. Wells, Approximating

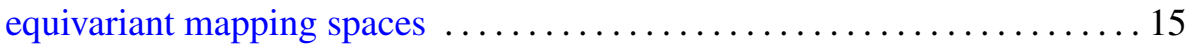

Peter Michael Higgins, A short proof of Isbell's zigzag theorem . . . . . . . 47

Harold H. Johnson, The absolute invariance of conservation laws ........5 51

Edgar Kann, Infinitesimal rigidity of almost-convex oriented polyhedra of arbitrary Euler characteristic $\ldots \ldots \ldots \ldots \ldots \ldots \ldots \ldots \ldots \ldots \ldots \ldots \ldots$

Alan Van Lair, Uniqueness for a nonlinear abstract Cauchy problem . . . . . 105

John B. Little and Kathryn A. Furio, On the distribution of Weierstrass points on irreducible rational nodal curves $\ldots \ldots \ldots \ldots \ldots \ldots \ldots \ldots \ldots 131$

J. S. Okon and Louis Jackson Ratliff, Jr., Reductions of filtrations . . . . . 137

Janusz Pawlikowski, Small subset of the plane which almost contains almost all Borel functions ............................... 155

Sergio A. Tozoni, Vector singular integral operators on a local field ....... 161 John Bason Wagoner, Triangle identities and symmetries of a subshift of

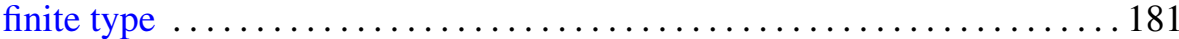

